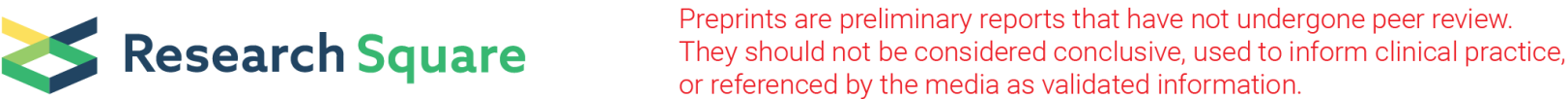

\section{FABP3 deficiency exacerbates metabolic derangement in cardiac hypertrophy via PPARa pathway}

\section{Lingfang Zhuang}

Ruijin Hospital, Shanghai Jiao Tong University School of Medicine https://orcid.org/0000-0003-27233202

\section{Zizhu Liu}

Ruijin Hospital, Shanghai Jiao Tong University School of Medicine

\section{Chenni Li}

Ruijin Hospital, Shanghai Jiao Tong University School of Medicine

\section{Qi Jin}

Ruijin Hospital, Shanghai Jiao Tong University School of Medicine

\section{Lin Lu}

Ruijin Hospital, Shanghai Jiao Tong University School of Medicine

\section{Rong Tao}

Ruijin Hospital, Shanghai Jiao Tong University School of Medicine

\section{Xiaoxiang Yan}

Shanghai Jiaotong University School of Medicine

Kang Chen ( $\nabla$ chenkang1978@163.com )

Ruijin Hospital, Shanghai Jiao Tong University School of Medicine

\section{Article}

Keywords: FABP3, cardiac hypertrophy, heart failure, energy metabolism, PPARa

Posted Date: February 5th, 2021

DOI: https://doi.org/10.21203/rs.3.rs-151701/v1

License: (c) (1) This work is licensed under a Creative Commons Attribution 4.0 International License. Read Full License 


\section{Abstract}

Cardiac hypertrophy was accompanied by various cardiovascular diseases (CVDs), due to the high global incidence and mortality of CVDs, it has become increasingly critical to characterize the pathogenesis of cardiac hypertrophy. We aimed to determine the metabolic effects of fatty acid binding protein 3 (FABP3), on transverse aortic constriction (TAC)-induced cardiac hypertrophy. TAC or Ang II treatment markedly upregulated Fabp3 expression. Notably, Fabp3 ablation aggravated TAC-induced cardiac hypertrophy and cardiac dysfunction. Multi-omics analysis revealed that Fabp3-deficient hearts exhibited disrupted metabolic signatures characterized by increased glycolysis, toxic lipid accumulation, and compromised fatty acid oxidation and ATP production under hypertrophic stimuli. Mechanistically, FABP3 mediated metabolic reprogramming by directly interacting with PPARa, which stabilized and regulated its transcriptional activity on Mlycd, Gck. Finally, treatment with the PPARa agonist, fenofibrate, rescued the pro-hypertrophic effects of Fabp3 deficiency. Collectively, these findings indicate that the FABP3-PPARa axis contributes to metabolic homeostasis and participates in the development of hypertrophy and heart failure.

\section{Introduction}

Cardiovascular diseases (CVDs) have become the primary cause of adult death worldwide ${ }^{1}$, cardiac hypertrophy is induced by various CVDs, such as hypertension, hypertrophic cardiomyopathy (HCM), and storage diseases (associated with abnormal accumulation of lipid, glycogen, and misfolded proteins), and it is accompanied by heart failure and arrhythmia, ultimately leading to death, highlighting the urgency for further elucidating the mechanism underlying the development of cardiac hypertrophy ${ }^{2}$. To date, studies have confirmed that numerous mechanisms contribute to the onset and progression of cardiac hypertrophy, such as increased cell death and fibrosis, impaired protein and mitochondrial quality control, and reprogrammed metabolism ${ }^{2,3}$. Of note, the role of metabolic rewiring in hypertrophic progression has more recently become a topic of research interest.

Through the use of advanced genomic technology, single-cell RNA sequencing (scRNA-seq), significantly transcriptional differences on cellular metabolism have been described as one of the most profound aspects contributing to cardiac dysfunction ${ }^{4}$. Specifically, an energy preference shifted from fatty acid boxidation (FAO) to glucose metabolism, with the downregulation of FAO genes and subsequently upregulation of glucose oxidation genes, has been described in pathophysiological conditions, such as hypertrophy, and heart failure ${ }^{5,6,7}$. Moreover, an increase in glucose consumption reportedly induced cardiac hypertrophy, while preservation of FAO improved myocardial energetics and cardiac function ${ }^{8,9}$.

FAO accounts for nearly $70 \%$ of ATP production in the postnatal heart, which underscores the pivotal role of fatty acid metabolism in maintaining heart function ${ }^{10}$. Unlike glucose, lipid species are insoluble and generally bind to lipid chaperones for transportation and utilization. Among them, fatty acid binding protein 3 (FABP3) is a small protein that is abundantly expressed in heart tissues and participates in cell metabolism by binding free long-chain fatty acids (LCFAs) and transporting them for cell metabolism, 
thereby protecting against lipid toxicity ${ }^{11}$. Additionally, FABP3 has been described in the context of cardiac hypertrophy, with a positive relationship being described between cellular and circulating levels of FABP3 and cardiac hypertrophy in patients and mice. However, the mechanism through which FABP3 affects cellular metabolic homeostasis and advances of cardiac hypertrophy remains poorly understood $^{12}$.

Accordingly, the present study aimed to determine the metabolic effects of FABP3 on transverse aortic constriction (TAC)-induced cardiac hypertrophy and heart failure using genetic mutant Fabp3-null mice. Our findings indicate Fabp3-defect exacerbates cardiac hypertrophy and heart dysfunction, resulting in defective FAO, and increased glycolysis by impairing the PPARa signaling pathway. Furthermore, the agonist of PPARa, fenofibrate, attenuated TAC-induced cardiac hypertrophy in both wild-type (WT) and Fabp3-null mice. Collectively, this study for the first time demonstrates the indispensable role of FABP3 on metabolic homeostasis and the advance of hypertrophy and heart failure.

\section{Results}

\section{Hypertrophic stimuli upregulate FABP3 expression}

First, to gain an overall profile of FABP3 expression, we examined its mRNA and protein level in mouse tissues, including the heart, liver, spleen, muscle, and brown adipose tissue (BAT), using quantitative polymerase chain reaction (qPCR) and western blotting assays. Our results showed that FABP3 protein and Fabp3 mRNA level was exclusively expressed in hearts, BAT and muscles, while its expression was rare in other organs, such as liver and brain, etc (Fig. 1a-c). Intriguingly, hearts and BAT have been recognized for their distinct preference for fatty acids as energy sources ${ }^{13}, 14$, which underscores the important role of FABP3 in cardiac fatty acid metabolism. Then, TAC operation was used to induce cardiac hypertrophy in vivo. By analyzing the expression of FABP3 at sham, 1-(1W), 2-(2W), 4-(4W), and 8weeks (8W) after TAC operation using qPCR and western blotting, we observed TAC operation induced FABP3 expression approximately 2-fold at $4 \mathrm{~W}$ compared with sham groups (Fig. 1d-f). Immunofluorescence co-staining of FABP3 and a-actinin showed a consistent increase of FABP3 expression after TAC treatment (Fig. 1g, h).

Next, to determine the expression of FABP3 in vitro following neurohormonal stimuli, neonatal rat ventricular myocytes (NRVMs) were treated with norepinephrine (NE) or angiotensin (Ang) II for $24 \mathrm{~h}$ and showed a significant increase of FABP3 levels using western blot. Moreover, Ang II induced higher FABP3 expression than NE (Fig. 1i, j). Therefore, we selected Ang II as the in vitro hypertrophic inducer for further experiments.

Next, we assessed the transcriptional expression of Fabp3 in scRNA-seq datasets, which analyzed the transcriptional differences in mice cardiomyocytes following sham operation or at 3 days (3D), 1W, 2W, $4 \mathrm{~W}$, or $8 \mathrm{~W}$ after TAC surgery, as well as in myocytes from dilated cardiomyopathy (DCM) and normal patients ${ }^{15}$. Similarly, scRNA-seq data showed significantly increased Fabp3 expression at 4W after TAC 
surgery compared to the sham group (Fig. 1k) and more than 5-fold higher Fabp3 expression in DCM patients than their normal counterparts (Fig. 11).

Taken together, these data suggest that FABP3 is expressed in heart tissues, which use fatty acids as a primary fuel substrate, and is upregulated in vivo and in vitro under hypertrophic stimuli.

\section{Loss Of FABP3 Aggravates Continuous Overload-induced Cardiac Hypertrophy}

To examine the effects of FABP3 on cardiac hypertrophy, we generated Fabp3 knock-out (F3-KO) mice using CRISPR/Cas9, the Fabp3-null mice were viable. Homozygous, heterozygous allele and wild type mice were identified using PCR (Supplementary Fig. 1a-c). Notably, cardiac FABP3 was completely abolished in Fabp3-null mice which allowed for a direct examination of the role of FABP3 on cardiac hypertrophy (Supplementary Fig. 1d, e).

To explore whether FABP3 contributes to TAC-induced hypertrophy, F3-KO and WT mice were subjected to TAC or sham surgery and observed for 4 weeks (Supplementary Fig. 2a). Firstly, to exclude the systemic differences among TAC-operated WT and F3-KO mice which may exert extra effects on the development of cardiac hypertrophy, organ mass to body weight ratio was measured and similar spleen, kidney, BAT, and white adipose tissue (WAT) ratio was found between WT and F3-KO mice, except for a higher brain weight in F3-KO mice (Supplementary Fig. 2b). Similarly, no difference was observed in liver mass among WT and F3-KO mice (Supplementary Fig. 2c). Moreover, hematoxylin and eosin (H\&E) staining of above organs showed no markedly structural differences between WT and F3-KO mice after TAC surgery, demonstrating that FABP3 deletion did not result in systemic abnormity after TAC operations (Supplementary Fig. 2d).

Next, to determine the effects of Fabp3-null on cardiac function, echocardiography (echo) was performed on WT and F3-KO mice at 4W after surgery (Fig. 2a). Compared with WT mice, TAC surgery led to severer cardiac hypertrophy in Fabp3-null mice, revealed as higher thickness of the interventricular septum (IVS; d: $1.56 \pm 0.03$ versus $1.17 \pm 0.04$; IVS; s: $2.02 \pm 0.05$ versus $1.63 \pm 0.03$; F3-KO versus WT) and left ventricular posterior wall thickness (LVPW; d: $1.38 \pm 0.08$ versus $1.11 \pm 0.06$; LVPW; s: $1.82 \pm 0.07$ versus $1.60 \pm 0.07$; F3-KO versus WT) in F3-KO mice, meanwhile these parameters were comparable under sham groups (Fig. 2b, c). Although no statistical difference was observed in the lung weight to body weight ratio (LW/BW) between WT and KO mice (Supplementary Fig. 2e), we found increased heart weight to body weight ratio (HW/BW) in F3-KO mice after TAC surgery compared with WT mice (Fig. 2d, $13.65 \pm$ 0.86 in F3-KO, $10.54 \pm 0.53$ in WT), this result together with the echocardiography data demonstrated a crucial role of $\mathrm{FABP} 3$ on cardiac hypertrophy.

Then, histological H\&E and WGA staining was conducted to determine the degree of cardiac hypertrophy after echo analysis. A significant increase in left ventricular to right ventricular wall thickness ratio (LV/RV) was found in F3-KO hearts compared to WT littermates after TAC surgery (Fig. 2e, f). Although no difference was observed in the cardiomyocyte area between sham group (139.35 \pm 4.86 in WT and $162.29 \pm 5.94$ in F3-KO), Fabp3-null caused a significantly enlarged cell area (595.64 \pm 15.44$)$ compared to the WT counterparts $(360.06 \pm 12.90)$ after TAC surgery (Fig. 2 g, h). Finally, hypertrophic genes, such as 
Anp, Bnp, Acta 1, and Myh7, were evaluated with qPCR assay. In line with the hypertrophic phenotype in Fabp3-KO hearts, the mRNA levels of Anp and Bnp were upregulated in F3-KO hearts after surgery compared to WT hearts (Fig. 2i). Similarly, ANP levels were increased by nearly 2-fold in F3-KO hearts compared as WT hearts (Fig. 2j, k). Altogether, we concluded that after TAC operation, FABP3 deficiency shows no effects on systemic abnormity, while contributes to the aggravation of cardiac hypertrophy.

\section{Loss Of FABP3 Impairs Cardiac Remodeling After Hypertrophy}

Cardiac hypertrophy contributes to heart failure under continuous overload, ultimately leading to adverse cardiovascular events and death. To determine whether Fabp3 deficiency is associated with hypertrophyinduced heart failure, we analyzed the mortality rate of WT and F3-KO mice over 8-weeks post-surgery. At the end of the observation period, a lower survival probability was observed in the F3-KO group compared to the WT group (Supplementary Fig. 3a, $p=0.3$ ). Then, the gross morphology of WT and F3-KO hearts was compared and showed increase of heart size in F3-KO mice after TAC surgery compared to WT hearts (Supplementary Fig. 3b). Consistent with enlarged hearts in Fabp3-defect mice, decline in left ventricular ejection fraction (LVEF, $27.58 \pm 1.52$ in F3-KO, $43.34 \pm 2.65$ in WT) and left ventricular fractional shortening (LVFS, $12.79 \pm 0.76$ in F3-KO, $21.27 \pm 1.52$ in WT) was found in F3-KO mice compared to their WT group at 8-weeks post-surgery, which was paralleled with higher left ventricular end diastolic volume (LVEDV) and left ventricular end systolic volume (LVESV) in Fabp3-null mice (Supplementary Fig. 3c-e). These results pointed to the important role of FABP3 on TAC induced cardiac hypertrophy and the progress of heart failure.

Then, considering that fibrosis serves as a hallmark of cardiac dysfunction, by measuring the level of fibrosis-related genes via qPCR assay, we found that the mRNA expression of Col3a 1 was increased in TAC-operated F3-KO hearts compared to WT hearts. Moreover, Fabp3 ablation reduced the mRNA expression of matrix metallopeptidase 2 (Mmp2) and matrix metallopeptidase (Mmp9) after TAC surgery, suggesting impaired collagen-turnover and homeostasis in Fabp3-KO mice (Supplementary Fig. 3f). In agreement with the increased expression of fibrosis genes in F3-KO hearts, Massons and Sirus red staining confirmed increased left ventricular collagen volume in F3-KO hearts (interstitial: $12.12 \pm 1.14$, perivascular: $7.28 \pm 0.45$ ) compared to WT hearts (interstitial: $4.83 \pm 0.57$, perivascular: $3.42 \pm 0.65$ ) (Supplementary Fig. 3g, h). Cumulatively, these data suggest that the loss of FABP3 contributes to compromised contractility and increased collagen volume following TAC operation.

\section{FABP3 alleviates Ang Il-induced cardiomyocyte hypertrophy in vitro}

To corroborate the above findings that FABP3 participates in TAC-induced hypertrophy, we manipulated the expression of Fabp3 in vitro using non-targeting small interfering RNA (siRNA) or lentivirus. The siRNA method reduced the mRNA level of Fabp3 by 70\% in H9C2 cells (Supplementary Fig. 4a). Firstly, NRVMs were transfected with siRNA-target Fabp3 (Si-F3) or its scrambled control (Si-NC) and the cell sectional area was measured after Ang II or PBS treatment. We showed cell size was comparable in the PBS groups, however, knocking-down Fabp3 significantly enlarged cell area after Ang II treatment 
compared with Si-NC group (Fig. 3a, b). Moreover, an increase of Anp and Bnp mRNA expression in line with upregulation of ANP protein level was found in Si-F3 group compared with Si-NC group (Fig. 3c-e).

Then, lentivirus vectors containing the full-length Fabp3 transcript (NM_001320996) and green fluorescent protein (GFP) were constructed, transfected and visualized with the fluorescence microscope to confirmed the transfection efficiency (Supplementary Fig. 4b). After transfecting H9C2 cells with optimal multiplicity of infection (MOI) of 10 and 100, lentivirus carrying the Fabp3 transcript (Lenti-F3) markedly increased FABP3 protein levels by more than 4-fold compared to the empty control vector (LentiCtl; Supplementary Fig. 4c, d), which was supported by higher FABP3 fluorescence value in the Lenti-F3 group (Supplementary Fig. 4e), these data confirmed the capability of lentivirus for upregulating the FABP3 expression. In contrast to the pro-hypertrophic effect of Fabp3 ablation, knocking-in expression of FABP3 prevented Ang II-induced cell hypertrophy in vitro. Specifically, Ang II stimulation resulted in enlarged cell sizes in control group, which was markedly blunted when overexpression of FABP3 (Fig. 3f, g). Consistent with the reduction in cell area, knocking in expression of FABP3 curbed the increase of Anp, $B n p$, and Myh7mRNA levels following Ang II treatment (Fig. 3h), as well as the protein expression of ANP (Fig. $3 \mathrm{i}, \mathrm{j}$ ). These in vitro data verified the protective effects of FABP3 on neurohormonal stimuli-induced hypertrophy. Altogether, these FABP3 loss- and gain-of-function results corroborate with in vivo phenotypes and reveal the important role of FABP3 in the development of cardiac hypertrophy.

\section{Ablation of FABP3 leads to defective FA b-oxidation and lipid homeostasis}

To explore the mechanism through which FABP3 regulates cardiac hypertrophy, we collected F3-KO or WT hearts at 1-week post-sham or -TAC operation for RNA-seq analysis and liquid chromatography-mass spectrometry (LC-MS) analysis to determine differential genes and metabolites (Fig. 4a). Principal component analysis (PCA) of RNA-seq revealed that the transcriptome of F3-KO hearts was similar to those of WT mice under sham conditions. However, TAC operation induced differentially expressed genes between WT and F3-KO hearts, which separated them on the PCA plot (Supplementary Fig. 5a). Comparing TAC-operated F3-KO hearts with WT hearts, a total of 939 (upregulated: 772, downregulated: 167) differentially expressed genes were identified and analyzed in this study (Fig. 4a). Further, KEGG pathway analysis revealed that these differentially expressed genes were enriched for terms related to "lipid metabolism," "glycan metabolism," and "energy metabolism" (Supplementary Fig. 5b).

Next, we performed gene set enrichment analysis (GSEA) based on GO biological process items using all detected genes. We found that "regulation of anatomical structure size," "wound healing," and "extracellular structure organization" were positively correlated with TAC-operated F3-KO mice. These pathways, which were consistent with pro-hypertrophic phenotypes in Fabp3-null hearts, suggested that ablation of Fabp3 trigger maladaptive remodeling after TAC operation (Supplementary Fig. 5c).

Intriguingly, GSEA analysis indicated that Fabp3 ablation caused a compromised mitochondrial fatty acid b-oxidation and lipid homeostasis. Specifically, "fatty acid beta oxidation using acyl-CoA dehydrogenase" was negatively correlated with F3-KO hearts (Fig. 4b, and Supplementary Fig. 5c), while positively enriched "lipid biosynthetic process" and "lipid storage" was found in the F3-KO hearts compared with 
WT hearts (Fig. 4c, and Supplementary Fig. 5d). In line with the aforementioned pathways, the suppressed FAO genes while activated lipid biogenesis genes were observed in F3-KO TAC group than WT TAC group in RNA-seq analysis and in vivo experiments (Fig. 4d, e). Specifically, malonyl-CoA decarboxylase $(M / y C d)$ which increases the rate of fatty acid oxidation, and carnitine palmitoyltransferase 1B (Cpt1b) was downregulated in F3-KO mice, meanwhile, acetyl-CoA carboxylase alpha (Acaca) which synthesis of malonyl-CoA results in immediate inhibition of fatty acid transport into mitochondria, Gpam and Agpat2, genes involve in the committed step in glycerolipid biosynthesis, were increased in F3-KO hearts in RNA-seq analysis and showed a consistent mRNA expression in vivo (Fig. 4d, e). However, these genes were comparable under sham conditions whether in RNA-seq analysis or their mRNA expression in vivo (Fig. 4d and Supplementary Fig. 5e), which manifested that Fabp3 deficiency has no different effect on cardiac energy metabolism under physiological conditions, however, hypertrophic stimulation that occurred with higher energy demand magnified the effects of Fabp3-null on energy homeostasis and resulted to abnormal FAO and lipid biosynthesis.

Furthermore, mitochondrial stress assay was performed in NRVMs with or without knocking-in expression of FABP3 in vitro to measure oxygen consumption rate (OCR). Compared with the negative control, overexpression of FABP3 resulted in significant increases of basal, maximal respiration, and spare respiratory capacity (Fig. 4f, g). Relative to glucose, fatty acid required more oxygen for its b-oxidation, therefore OCR servers as a relative indicator of cell fuel preference. Higher OCR rate in lenti-F3 group was mirrored with the lowed expression of FAO genes in F3-KO hearts, indicating overexpression of FABP3 increased FAO. Then, supplement of etomoxir was used to evaluate the cell dependency on fatty acid as energy substrate. We found that application of etomoxir lead to a markedly drop of respiration in FABP3 knocking-in group compared with its negative control groups, demonstrating NRVMs with FABP3 overexpression have increased reliance on fatty acid as energy fuel (Fig. 4h, i). Moreover, impairing FAO with etomoxir would reverse the protective effects of $\mathrm{FABP} 3$ on cardiac hypertrophy and upregulated the mRNA expression of Bnp and Anp (Supplementary Fig. 5f). Taken together, these data points to the dependency of FABP3 on cardiomyocyte FA b-oxidation to meet an effective metabolic demand under hypertrophic stimulations.

\section{FABP3-null Hearts Exhibit Abnormal Lipid Accumulation}

As the RNA-seq analysis showed that FABP3 deletion led to abnormal transcriptional profile of FAO and lipid biogenesis (Fig. 4b-d). Lipid-targeted metabolomics analysis was used to determine the differential metabolites in WT and F3-KO hearts. We found that rather than triglycerides (TAG) and Acyl-carnitine (ACar), the fatty acid (16:0) and diglyceride (DAG 18:2-22:5, 18:2-18:2) were significantly increased in F3-KO hearts compared with WT mice (Fig. 4j, k and Supplementary Fig. 6b, c), which was in line with an increase of neutral lipid in Fabp3 knocking-downing cells after Ang II treatment (Supplementary Fig. 6a). It is important to note that previous researches showed that DGA and saturated fatty acid, especially palmitate, was associated with cellular toxicity for their direct actions as signaling lipids ${ }^{16}$. Therefore, FABP3 deficiency contributed to accumulation of toxic lipids in hearts under hypertrophic stimuli. Consistent with increased toxic lipid species and neutral lipid in vivo and in vitro, we observed a higher 
level of lipid droplet accumulation in Fabp3-null hearts by using transmission electron microscopy (TEM), which was rare in WT hearts (Fig. $4 \mathrm{l}$ and Supplementary Fig. 6d). To determine whether differences in lipid uptake accounted for the abnormal lipid accumulation in F3-KO mice, plasma non-esterified fatty acid (NEFA) was measured at sham, $4 \mathrm{~W}$ and $8 \mathrm{~W}$ post-surgery, we found that the plasma concentration of NEFA was higher at 8W compared to the sham group; however, no significant difference was observed between WT and F3-KO mice (Supplementary Fig. 6e), this data in line with comparable mRNA expression of fatty acid transporter $C d 36$ between WT and F3-KO hearts (Fig. 4d, e) revealed that differences in lipid uptake did not responsible for the greater severity of tissue lipid accumulation in F3-KO mice. Taken together, we showed that Fabp3-null contributes to defective FAO, which triggered the accumulation of long-chain FAs, especially palmitate in F3-KO hearts, and excessive incorporation of DAG and other lipid subspecies, eventually resulted in an increased accumulation of toxic lipid in F3-KO hearts (Supplementary Fig. 6f).

\section{FABP3 Defect Hearts Show Increased Reliance On Glycolysis}

In the context of diminished capacity for FAO, we next sorted to determine whether shifted chief energy substrate from fatty acids to glucose was found in F3-KO hearts. Intriguingly, GSEA analysis revealed that the loss of FABP3 triggered abnormally activated glucose metabolic pathways, such as "glucan catabolic process" (Supplementary Fig. 7a) and "regulation of gluconeogenesis" (Supplementary Fig. 7b). In line with activated glucose oxidation and gluconeogenesis pathways, glucokinase (Gck), a gene that participates in the first step in glucose metabolism, 6-phosphofructo-2-kinase/fructose-2,6-biphosphatase 2 (Pfkfb2), and phosphoenolpyruvate carboxykinase 1 (Pck1) was upregulated in F3-KO TAC hearts (Fig. 5a). A consistent mRNA expression profile in vivo confirmed that increased Gck and Pck1 in Fabp3 deficiency hearts after TAC surgery (Fig. 5b). Meanwhile, "mitochondrial electron transport NADH to ubiquinone" was negatively correlated with F3-KO hearts, which was consistent with markedly dropped expression of TCA cycle genes (Ogdh, Idh2, Aco2) in TAC-operated Fabp3-null hearts than in WT mice, indicating reduced mitochondrial TCA cycle, OXPHOS and ATP production in Fabp3-null hearts under hypertrophic stress (Fig. 5a and Supplementary Fig. 7c). To determine whether Fabp3-defect increases glucose uptake further activates glucose oxidation, we found the glucose transporter, solute carrier family 2 member 1 (S/c2a 1) and solute carrier family 2 member 4 (S/c2a4) was downregulated in F3-KO hearts compared with WT mice (Fig. 5a, b), these results were consistent with normal serum glucose, similar glycogen content in small intestine and live, suggesting that Fabp3-defect had no role on the uptake of glucose during cardiac hypertrophy (Supplementary Fig. 7d, e).

Based on activated glucose oxidation genes, we next performed gas chromatography -mass spectrometry (GC-MS) analysis to quantify differential metabolites in WT and F3-KO hearts after TAC surgery. PCA analysis of these metabolites showed that F3-KO hearts were separated from WT samples, which was consistent with the OPLS-DA analysis results (Supplementary Fig. 7f, g). Furthermore, KEGG pathway analysis of differential metabolites indicated that "arginine and proline metabolism" and "citrate cycle" was significantly enriched in F3-KO hearts (Supplementary Fig. 7h). Additionally, we observed significantly increased glycolysis pathway related-metabolites (glucose, glucose-6-phosphate, and 
fructose 2,6-biphosphate) and considerably reduced TCA cycle metabolites (malic and fumaric acids) in Fabp3-deficient hearts as compared to WT hearts (Fig. 5c). furthermore, by measuring the concentration of glucose-6-phosphate (G6P), an indicator of cellular glycolytic flux, we showed a higher concentration of G6P in Fabp3-knocking down cells, however, overexpression of Fabp3 resulted in dramatic decline in the G6P level (Fig. 5d), which manifesting a glycolytic shift on fuel substrate when knocking-down expression of Fabp3.

Based on the results from the RNA-seq and metabolomics, we performed a glycolytic rate assay to realtime analyze cellular glycolysis by calculating proton efflux rate from glycolysis (glycoPER), a parameter that measures acidification from glycolysis without any contribution from mitochondrial respiration. We observed that NRVMs with Fabp3 knocking-down exhibited a higher basal and compensatory glycolysis compared to its negative control after Ang II stimulation, however, the glycolytic rate showed no significant differences between these two groups under PBS treatment (Fig. 5e, f). In striking contrast to increased glycolysis in Fabp3 knocking-down cells, overexpression of Fabp3 resulted in a marked drop of glycolysis than the control cells after Ang II treatment (Fig. $5 \mathrm{~g}, \mathrm{~h}$ ). All results combined with multi-omics analysis and glycolytic energetics demonstrated that Fabp3 deficiency resulted in a shift in glycolysis as fuel source and compromised TCA and ATP production (Fig. 5i).

Taken together, these observations confirm that in addition to compromised FAO and ATP production, loss of FABP3 increases glycolysis and toxic lipid accumulation, both of which ultimately aggravate metabolic derangement and heart failure.

\section{FABP3 mediates PPARa level by binding and stabilizing PPARa, further enhances its transcriptional activity under hypertrophic stimuli}

As mentioned before, Fabp3 deficiency contributes to deranged metabolic milieu characterized by reduced FAO and increased glycolysis. Next, we sought to explore the mechanism through which Fabp3 mediates metabolic derangement during cardiac hypertrophy. Firstly, our RNA-seq analysis verified that the "PPAR signaling pathway" was one of the top enriched pathways in Fabp3-deficient hearts (Supplementary Fig. 8a). Definitive evidence supports the critical requirement for peroxisome proliferatoractivated receptor (PPAR), particularly PPARa, in myocyte metabolism and metabolic reprogramming under cardiac hypertrophy ${ }^{17}$. Therefore, we postulated that FABP3 participates in cellular metabolism through the PPARa signaling pathway. Firstly, to delineate the mRNA and protein level of PPARa in Fabp3defect hearts, we found that the mRNA expression of Ppara showed no difference in WT and F3-KO heart (Supplementary Fig. 8b), However, its protein expression was marked downregulation in Fabp3 deficiency mice as compared to WT hearts following TAC surgery. Conversely, in vitro overexpression of Fabp3 using a lentivirus vector rescued the protein level of PPARa following Ang II stimulation (Fig. 6a, b). Immunofluorescence staining of PPARa at $4 \mathrm{~W}$ post-surgery confirmed that TAC operation resulted in decrease and perinuclear shuttling of PPARa, while Fabp3 ablation accelerated its loss (Fig. 6c, d). These findings indicate that FABP3 might participate in metabolic homeostasis during cardiac hypertrophy via 
PPARa signaling. However, the mechanism through which FABP3 targets PPARa for metabolic regulation remains elusive.

Next, we transfected NRVMs with Fabp3 or Ppara, co-immunoprecipitated with respective antibodies, and analyzed using SDS-PAGE, the results showed that FABP3 directly bond with PPARa, with or without Ang II stimulation (Fig. 6e, f). As we have shown before, Fabp3 deletion exerted no effect on Ppara mRNA level, suggesting a post-transcriptional modification role of FABP3 on the protein level of PPARa. Then, the NRVMs with Fabp3 knocking-in were treated with cycloheximide (CHX) to eliminate protein translational regulation. We found that Fabp3 overexpression markedly prolonged the half-life of PPARa compared with its negative control, suggesting FABP3 increased PPARa protein level by inhibiting its degradation (Fig. 6g, h). Finally, to determine whether the interaction of FABP3 with PPARa affected its transactivation, we performed luciferase gene transactivation assays in HEK 293T cells. The activation of PPARa was determined based on a reporter plasmid containing firefly luciferase after three PPARresponsive-element (PPRE 3 -TK-LUC). Following co-transfection of 293T cells with PPARa, FABP3, and $\mathrm{PPRE}_{3}$-TK-LUC, we found PPARa significantly increased the luciferase expression of PPRE ${ }_{3}$-TK-LUC; moreover, co-transfection of FABP3 and PPARa induced higher PPRE-driven luciferase activity compared with PPARa alone (Fig. 6i). Together, these findings indicate that FABP3 mediates the protein level of PPARa by interacting with PPARa, inhibiting its degradation, and modulating its transcriptional activity during cardiac hypertrophy.

\section{Required of PPARa on FABP3-modulated FAO/glycolysis balance and cardiac hypertrophy}

As the pleiotropic effects of PPARa on inhibiting glucose oxidation, while activating FAO, we aimed to determine whether FABP3 interacts with PPARa and modulates its transcriptional capacities on $\mathrm{FAO} /$ glycolysis genes and further involves in the advance of cardiac hypertrophy. Firstly, we curated PPARa target genes in our RNA-seq to reveal their transcriptional profile in sham or TAC operated-WT and F3-KO hearts (Supplementary Table. 1). We observed that the transcriptional level of Gck (participated in glycolysis) and Acaca (participated in lipogenesis) was upregulated in F3-KO hearts, while Mlycd and $C p t 1 b$ (participated in mitochondrial FAO) was significantly reduced compared to WT hearts. The western blot assay confirmed a similar protein expressional pattern of MLYCD, CPT1B, ACC, GCK in Fabp3-null hearts. Specifically, Fabp3-ablation led to lower MLYCD and CPT1B protein level while upregulated ACC and GCK, suggesting a direct effect of FABP3 on MLYCD and CPT1B, and an inverse transcriptional effect of FABP3 on ACC and GCK via PPARa (Fig. 6j, k). Therefore, to address the question of whether FABP3 participated in the transcriptional activation of FAO and glycolysis genes via PPARa. We constructed reporter plasmid containing firefly luciferase and Mlycd or Gck promoter (Mlycd-LUC, Gck-LUC, respectively), after transfecting 293T cells with PPARa, FABP3, Mlycd-LUC or Gck-LUC, we showed that PPARa increased Mlycd transcriptional activity; however, higher luciferase activity was observed when 293T cells were co-transfected with FABP3 and PPARa (Fig. 6l). In contrast to Mlycd-LUC, PPARa blunted the transactivation of Gck-LUC, which showed a severer inhibition in the presence of FABP3 (Fig. $6 \mathrm{~m}$ ). These data suggest the metabolic regulatory role of FABP3 in transcriptional activating FAO genes Mlycd, Cpt1b and curbing glycolysis and lipogenesis genes Gck, Acaca via PPARa (Fig. 6n). 
Next, to demonstrate the requirement of PPARa in FABP3 mediated cardiac hypertrophy, PPARa was knocked down using siRNA methods as described previously (Supplementary Fig. $8 \mathrm{c}, \mathrm{d}$ ) ${ }^{18}$. NRVMs with knocking-in expression of FABP3 were transfected Si-PPARa or its scrambled control, we observed that PPARa downregulation abolished the protective effects of FABP3 on cardiomyocyte hypertrophy, resulted in enlarged cell area (Supplementary Fig. 8e, f) and upregulation of Bnp and Anp (Supplementary Fig. 8g). Notably, increased cellular neutral lipid was found after knocking-down PPARa (Supplementary Fig. 8h). Altogether, these data illustrate that FABP3 participates in cardiac hypertrophy by synergistically activating Mlycd, Cpt1b and repressing Gck, Acaca via PPARa.

\section{Activation of PPARa with fenofibrate reverses FABP3-KO induced cardiac hypertrophy}

As observed previously, Fabp3 deficiency contributes to hypertrophy and deranged metabolic milieu by impairing PPARa pathway. We next sought to determine whether activating PPARa may rescue the prohypertrophic effects of Fabp3-defect after TAC operation and search for clinical benefits on the treatment of cardiac hypertrophy. Firstly, NRVMs were treated with fenofibrate, a PPARa-specific agonist, or vehicle (DMSO) for $24 \mathrm{~h}$, and consistent with previous results, knock-down of Fabp3 with siRNA (Si-F3) resulted in increased cell cross-sectional area compared to its control (Si-NC), while fenofibrate treatment markedly reduced the cell area in both the Si-NC and Si-F3 groups (Fig. 7a, b).

Next, PPAR agonist studies were performed in vivo to investigate the effects of fenofibrate on Fabp3defect-induced hypertrophy. WT and Fabp3-KO mice were subjected to TAC surgery and randomly treated with fenofibrate $(100 \mathrm{mg} / \mathrm{kg} / \mathrm{d})$ or vehicle by oral gavage daily for $4 \mathrm{~W}$. Interestingly, compared with the vehicle group, fenofibrate treatment significantly rescued cardiac hypertrophy in WT and Fabp3-KO mice. More specifically, IVS and LVPW were markedly decreased following fenofibrate treatment (Fig. 7c-e). Consistent with attenuated cardiac function, hypertrophic and fibrosis-related genes, such as Anp, Bnp, and Col3a1, were inhibited by fenofibrate treatment, but not by the vehicle (Fig. 7f). In addition, histochemical analysis showed that Fabp3 deletion increased left ventricular wall thickness, cardiomyocyte size, and collagen volume, while fenofibrate supplement significantly rescued these effects whether in WT or Fabp3-KO hearts (Fig. 7g-I).

Collectively, these results demonstrate an important role for the PPARa pathway in Fabp3-KO-induced cardiac hypertrophy, while treatment with fenofibrate may reverse hypertrophy, suggesting potential clinical value for fenofibrate in hypertrophic treatment.

\section{Discussion}

Herein, we observed an indispensable role of FABP3 in the incidence and advance of cardiac hypertrophy. Fabp3 deficiency served to exacerbate TAC-induced hypertrophy and cardiac dysfunction, while its overexpression rescued cardiomyocyte hypertrophy in vitro. Mechanistically, Fabp3 defect resulted in metabolic derangement characterized by defective FAO and increased glycolysis to elicit imbalanced FAO/glycolysis rate and toxic lipid accumulation. Furthermore, Fabp3 mediated the PPARa protein levels 
by directly binding to PPARa and preventing its degradation and underpinning its transactivation on Mlycd and Gck, which underscores the pivotal metabolic role of PPARa in FABP3-mediated cardiac hypertrophy. Additionally, treatment with the PPARa agonist, fenofibrate, effectively repressed Fabp3-KO induced cardiac hypertrophy, highlighting a potential clinical value of hypertrophic treatment by targeting cardiac energy metabolism (Fig. 8).

FABP3, a small molecular weight protein, is abundantly expressed in heart and muscle tissues ${ }^{19}$. In our previous studies, we reported that FABP3 plays an important role in myocardial infarction and in-stent restenosis ${ }^{20,21}$. Moreover, clinical studies have considered FABP3 to be a marker of cardiac ischemic injury, demonstrating that its expression is associated with major adverse cardiac outcomes and recurrent $\mathrm{ML}^{22}, 23,24,25,26$. Emerging articles point to increased FABP3 expression is observed under cardiac hypertrophy, they demonstrate that a tightly control of FABP3 level by IGF-1 and miR-1. Under hypertrophic stimulations, myocardium shifts for higher energy demand which occurs with upregulation of IGF-1 and decline of miR-1, exposure to IGF-1 results to increase FABP3 expression, on the other hand, miR-1 downregulation able release its inhibitory role on FABP3 and eventually, elicit the increase of FABP3 under cardiac hypertrophy ${ }^{12}$. In line with these effects, we showed FABP3 was increased under hypertrophic stimulations in vivo and in vitro, moreover, FABP3 deficiency aggravates TAC induced cardiac hypertrophy and heart failure. To the best of our knowledge, this is the first study to explore the role of FABP3 during cardiac hypertrophy.

FABP3 can directly bind to cellular insoluble LCFAs and transport them to the mitochondrion, nucleus, or endoplasmic reticulum for utilization ${ }^{11}$. Notably, single-cells RNA-sequencing research suggested that the increased expression of Fabp3 from embryo to mature cardiomyocytes accounts for the developmental metabolic switch from embryonic glycolysis to postnatal mitochondrial fatty acid oxidation, underscoring its profound effects on fuel preference and cardiac metabolism ${ }^{27}$. Besides that, the loss- and gainfunction of FABP3 in brown adipocytes shows that FABP3 is a determinant of cellular fatty acid oxidation efficiency, brown adipocytes without FABP3 show defective capability to oxidize exogenously supplied fatty acids ${ }^{28}$. This leads to the intriguing question whether FABP3 participates in the development of cardiac hypertrophy by modulating cardiac metabolic homeostasis, especially the FAO and glucose oxidation processes. Here, we combined multi-omics analyses, such as RNA-seq and metabolomics, to reveal the indispensable role of FABP3 in governing cardiac energy regulatory program by increasing FAO and inhibiting glycolysis under hypertrophic stimuli.

As we have shown, Fabp3 deletion contributes to dramatic abnormalities in myocardial metabolism by targeting PPARa pathways. Accumulating evidence has indicated that PPAR family members (PPARa, $\mathrm{b} / \mathrm{s}$, and g), a member of nuclear receptor superfamily of transcription factor (NR1C), play regulatory roles in cellular metabolism and cardiac hypertrophy, which was confirmed in loss- or gain-of-function mutated mice ${ }^{29}$. Specifically, PPAR can directly bind to the promoter regions of metabolic-related genes and regulate their transcriptional levels ${ }^{30}$. PPARg is primarily expressed in adipose tissues, while PPARb/s is ubiquitously expressed. Of note, PPARa is exclusively expressed in tissues with higher capacity for fatty

Page $12 / 34$ 
acid oxidation, such as the heart, liver, and BAT, which display a concordant expression profile with FABP3 ${ }^{31}$. Cardiac-specific overexpression of PPARa resulted in activation in FA transport genes and suppression of glycolytic genes, however, these metabolic phenotypes were strikingly contrasted to cardiac-specific overexpression of PPARb/s which shows the increase of glycolysis and glucose uptake genes, indicating the reciprocal role of PPARa and PPARb/s on the regulation of cardiac metabolic homeostasis ${ }^{32}$. Based on the expression profile and metabolic effects, the interactive effects of FABP3 and PPARa in cellular metabolism and cardiac hypertrophy were explored in this article. Previous researches show that activation of PPARa exacerbates the uptake and utilization of FA and reduces glucose utilization $33,34,35$. Fasting or inhibiting mitochondrial FAO with etomoxir leads to markedly increased lipid accumulation in PPARa-/- heart and hepatocytes ${ }^{17}$. These phenotypes were similar to the derangement FAO/glucose oxidation rate in our FABP3-deficient mice, corroborating a link between FABP3 and PPARa. Moreover, studies have demonstrated a direct interaction between PPARa and FABP family members, especially FABP1 and FABP4, reporting that FABP1 or FABP4 translocate from the plasma to the nucleus, where it binds to PPARa to stabilize and amplify its biological function by enhancing its transcriptional activities ${ }^{36,37,38}$. Additionally, a luciferase assay containing FABP3 and PPARa plasmid suggests that FABP3 facilitates the transcriptional activity of PPARa in COS-7 cells ${ }^{39}$. However, whether FABP3 could interact with PPARa and governs its transcriptional activity in cardiomyocytes during chronic hypertrophic stimulation has not been fully delineated. Here, our in vivo and in vitro results reveal the potential mechanism of FABP3-mediated PPARa pathway in cardiac hypertrophy and in FAO/glycolysis balance by directly binding to PPARa, promoting its stability, underpinning its transactivation in Mlycd and Gck.

Collectively, our study provides novel insights into the regulatory role of FABP3 on cellular metabolism following TAC-induced cardiac hypertrophy. Meanwhile, targeting FABP3 with agonist may represent an attractive approach to alleviate deranged metabolic milieu in cardiac hypertrophy and achieve the goal of improved heart function.

\section{Methods}

\section{Animals and generation of FABP3-KO mice}

C57BL/ 6 male mice were purchased from SLAC Laboratory Animal Co., Ltd (Shanghai, China). Global Fabp3 knock-out mice were generated using the CRISPR-Cas9 method as described previously ${ }^{21}$ and housed in cages at room temperature and a $12 \mathrm{~h}$ light/dark cycle. All animal experimental procedures were approved by the Animal Care Committee of Shanghai Jiao Tong University School of Medicine.

\section{Transverse aortic constriction (TAC) Model}

The TAC model was used to induce pathological cardiac hypertrophy in vivo as described previously ${ }^{40}$. Briefly, 8-week-old mice were anesthetized with isoflurane, intubated, and mechanically ventilated with a low concentration of isoflurane gas (1.0\%). After Opening the sternum and moving aside the thymus 
glands, the aortic arch was visualized and ligated with $6-0$ silk suture against a 27-gauge needle, then removed the needle and closed the chest with $5-0$ silk suture. Mice were observed after 15 min for recovery. Sham-operated mice underwent an identical procedure except for aortic constriction.

Western blotting analyses: Total protein extracted from mice organs and cultured cells using SDS lysis (50 mM Tris, pH8.1, 1\% SDS, sodium pyrophosphate, $\beta$-glycerophosphate, sodium orthovanadate, sodium fluoride, EDTA, and leupeptin) were used for western blot analysis as described previously ${ }^{21}$. Antibodies against the following antigens were used: FABP3 (LS-C172162, LSBio, Seattle, WA, USA), GAPDH (97166, CST, Danvers, MA, USA), a-TUBULIN (66031-1-lg, Proteintech, Wuhan, Hubei, China), PPARa (sc-398394, Santa Cruz, Dallas, Texas, USA), ANP (sc-515701, Santa Cruz, Dallas, Texas, USA), MLYCD (15265-1-AP, Proteintech, Wuhan, Hubei, China), CPT1B (22170-1-AP, Proteintech, Wuhan, Hubei, China), ACC (67373-1Ig, Proteintech, Wuhan, Hubei, China), GCK (19666-1-AP, Proteintech, Wuhan, Hubei, China). GAPDH and aTUBULIN were used as controls. Densitometry values were measured by ImageJ for further analysis.

\section{Real-time quantitative polymerase chain reaction (RT-qPCR)}

Total RNA from mouse organs and cultured cells was extracted using Trizol reagent according to the manufacturer's protocols (Thermo Fisher Scientific Co., CA, USA). Primers for specific genes are presented in Supplementary Table. 2. The mRNA expression of indicated genes was amplified with ChamQ Universal SYBR qPCR Mix Kit (Q711-02, Vazyme, China) and detected using the Applied Biosystems QuantStudio 6 Flex Real-Time PCR System. The relative levels of specific genes were calculated by normalizing the level of Gapdh or $18 S$ using the $2^{-\Delta \Delta C T}$ method.

Immunocytochemistry and immunofluorescence staining (IF): After antigen retrieval and permeabilization, cultured cells or heart sections were blocked with 5\% BSA buffer for 30 min, following which the heart samples were probed overnight with primary antibodies. After washing with PBS and incubating with corresponding Alexa Fluor-conjugated secondary antibodies for 2 hours and DAPI for 5 min, the images were visualized using fluorescence microscopy and analyzed with ImageJ. Antibodies against the following antigens were used for IF staining: FABP3 (LS-C172162, LSBio, Seattle, WA, USA), PPARa (sc-398394, Santa Cruz, Dallas, Texas, USA), a-actinin (A7811, Sigma, St. Louis, MO, USA), and CTnT (GB11364, Servicebio, China). Quantification was performed using a minimum of three individual samples for each group, and a minimum of five high-resolution images were acquired for each sample.

\section{Echocardiography}

Cardiac function was measured at 4 or 8 weeks after surgery using the Vevo 2100 High-Resolution Digital-Imaging System (Visual Sonics). After being anesthetized with $3 \%$ isoflurane, the mice were restrained on the warmed platform without isoflurane to obtain higher heart rates. The ventricular $M$ and B-mode ultrasound at the papillary muscle level was measured to determine the heart size, thickness of the ventricular wall and septum, and evaluate cardiac function. All echo images captured and calculated were blinded to the researchers. 


\section{Histological analysis}

Mice were anesthetized using isoflurane and transcardially perfused with PBS and 4\% paraformaldehyde sequentially. Tissues were fixed overnight in paraformaldehyde and then embedded in paraffin or optimal cutting temperature compound (OCT) for histology and IF analysis. Standard hematoxylin and eosin (H\&E) staining was performed for morphological comparison. The volume of ventricular collagen was assessed by Masson's trichrome staining and Sirius red staining using ImageJ. Notably, images of Sirius red staining were captured with fluorescence microscopy according to a previously described protocol ${ }^{41}$. All experiments were performed at least in triplicate for achieving statistical significance.

Cardiomyocyte cross-sectional area analysis: Heart sections or cultured cells were stained with Wheat Germ Agglutinin (WGA, W11261, Invitrogen, Carlsbad, CA, USA) or a-actinin (A7811, Sigma, St. Louis, MO, USA) to determine the relative cell area in vivo and in vitro, respectively. The procedures of a-actinin staining were similar to IF assay as described previously. For WGA staining, heart sections were incubated with WGA working buffer (1:200) diluted in PBS for 20 min at room temperature after dewaxing and rehydration, following which the images were captured with fluorescence microscopy. To compare the myocyte cell area, images were acquired from at least three samples and 100 cells in hearts tissue and 50 cells in cultured dishes were measured per group using ImageJ.

\section{Neonatal rat ventricular cardiomyocyte (NRVM) isolation}

NRVMs were isolated from 1 to 3-day-old Sprague Dawley rats according to our lab protocols. In brief, hearts were cut into 1-3 mm pieces and digested with type II collagenase $(0.2 \mathrm{mg} / \mathrm{mL}$, Worthington Biochemical Corp, Freehold NJ, USA) diluted in HBSS buffer. After gently shaking at $37^{\circ} \mathrm{C}$ for 7 min for $3-$ 5 times, cardiomyocytes were released and plated with the cultured medium for $1.5 \mathrm{~h}$ to separate myocytes and fibroblasts. The myocytes were then re-plated with the culture medium (10\% FBS, DMEM/F12, $1 \%$ penicillin/streptomycin) for 24 hours in a humidified atmosphere with $5 \% \mathrm{CO}_{2}$ at $37^{\circ} \mathrm{C}$.

\section{Lentiviral constructs and transduction}

cDNA including the whole Fabp3 gene (NM_001320996.1) was amplified via PCR, verified by sequencing, and cloned as a positive plasmid (LV-EF1a $>$ FABP3/FLAG-CMV > eGFP/T2A/Puro). The negative control plasmid contained similar fragments as the positive control, while lacked the Fabp3 element (LV-CMV > eGFP/T2A/Puro). H9C2 cells or NRVMs were transfected with lentivirus at an optimal multiplicity of infection of 10 , and incubated with polybrene $(5 \mu \mathrm{g} / \mathrm{mL})$ for at least 24 hours, followed by selection with puromycin. The plasmid contained green fluorescent protein (GFP) allows for visualization under fluorescence microscopy.

\section{Bulk RNA sequencing (RNA-seq)}

WT and F3-KO hearts were collected at 1-week post-sham or TAC surgery and immediately frozen in liquid nitrogen. Total RNA was extracted from above tissues using Trizol according to standard protocols. 
Bioinformatics and significance analyses were performed in triplicate for statistical significance. Procedures of RNA-seq analysis, including RNA extraction, quantification, and cDNA library preparation, were performed by BGI Genomics procedures (BGI-SHENZHEN, China), using standard and consistent procedures.

RNA-seq data analysis: Sequencing data were filtered with SOAPnuke (v1.5.2) ${ }^{42}$ to remove reads containing sequencing adapters and those with more than $1 / 5$ low-quality base ratio. The resulting clean reads were saved in FASTQ format. The transcriptional reads were mapped to the reference genome using HISAT2 (v2.0.4) ${ }^{43}$ and STAR (v2.3.0) ${ }^{44}$. To identify differentially expressed genes, the expression of all mapped genes was calculated and analyzed based on their respective fragments per kilobase million (FPKM) value using the DESeq2 (v1.4.5) ${ }^{45}$ with $\mathrm{p}$ value $\leq 0.05$. Gene ontology (GO) (http://www.geneontology.org/) and KEGG (https://www.kegg.jp/) enrichment analyses of differentially expressed genes were performed using Phyper based on the hypergeometric test. The significant levels of terms and pathways were corrected by $Q$ value with a rigorous threshold ( $Q$ value $\leq 0.05$ ) by Bonferroni's test. Gene set enrichment analysis (GSEA) based on c5: GO gene sets dataset downloaded from the official website was performed ${ }^{46}$. Genes related to fatty acid oxidation, glucose metabolism, TCA cycle, and lipid biogenesis were manually selected and plotted as the heatmap using all sample expression matrix in Rstudio with the package Pheatmap.

\section{Transmission Electron Microscopy (TEM)}

After perfusing with cold PBS, the left ventricles were dissected into 1-2 $\mathrm{mm}$ pieces, fixed immediately in $2.5 \%$ glutaraldehyde buffer at room temperature for 2 hours, and then stored overnight at $4^{\circ} \mathrm{C}$. A $70-90$ $\mathrm{nm}$ thin section was cut and imaged using TEM. All TEM images were obtained by technicians that were blinded to the study design.

Metabolite extraction and gas chromatography-mass spectrometry (LC/GC-MS) analysis: WT and F3-KO mice were subjected to TAC surgery, after which their hearts were dissected at 1-week post-surgery and immediately frozen in liquid nitrogen. Approximately $50 \mathrm{mg}$ of tissue was added to an extraction mix (methanol: water: chloroform = 6: 3: 4), vortexed for $30 \mathrm{~s}$, homogenized for $4 \mathrm{~min}$, and ultra-sonicated for $5 \mathrm{~min}$ followed by centrifugation at 10,000 rpm for $15 \mathrm{~min}$. After evaporation in a vacuum concentrator, all samples were analyzed using an Agilent 7890 GC-MS with a DB-5MS capillary column. Metabonomic analysis, including metabolite extraction and GC-MS analysis, annotation, and pathway enrichment analysis were performed by Shanghai Biotree Biomedical Technology Co., Ltd. according to standard procedures.

GC-MS data annotation and analysis: The GC-MS raw data analysis, including peak extraction, baseline adjustment, deconvolution, alignment, and integration, was completed using the Chroma TOF (V 4.3x, LECO) software. The LECO-Fiehn Rtx5 database was used for metabolite identification by matching the mass spectrum and retention index. Finally, the peaks detected in less than half of the QC samples were removed ${ }^{47}$. After data management and normalization, approximately 478 peaks were included. First, 
principal component analysis (PCA) and orthogonal projections to latent structures-discriminant analysis (OPLS-DA) ${ }^{48}$ was performed to compare the group variances in SIMCA software (V15.0.2, Sartorius Stedim Data Analytics AB, Umea, Sweden $)^{49}$. Next, differential metabolites were identified with $p$ value < 0.05 using Student's $t$-test and variable importance in the projection (VIP) of OPLS-DA score $>1$. In addition, commercial databases including KEGG pathway and MetaboAnalyst (http://www.metaboanalyst.ca/) were used for pathway enrichment analysis.

\section{Plasma non-esterified fatty acid (NEFA) assay}

To quantify the concentration of NEFA in mouse serum, the NEFA assay was performed according to the manufacturer's protocols (294-63601, Wako, Japan). Briefly, blood obtained from sham or TAC-operated mice was rested at room temperature for 2 hours followed by centrifugation ( $2000 \mathrm{~g}, 20 \mathrm{~min}$ ), after which the plasma was collected. Approximately $4 \mu \mathrm{L}$ of serum was added to each well of the 96 -well microplate and mixed with the reaction solution. Absorbance was then measured at $550 \mathrm{~nm}$ with a spectrophotometer.

\section{co-Immunoprecipitation (co-IP)}

NRVMs with a knocking-in expression of Fabp3 or Ppara were treated with Ang II for $24 \mathrm{~h}$. Cultured cells were then homogenized in IP/lysis buffer (50 mM Tris, $150 \mathrm{mM} \mathrm{Nacl,} \mathrm{1 \%} \mathrm{NP-40,} 1$ mM EDTA, PH 7.4) supplemented with PMSF and phosphatase inhibitor cocktails (Sigma-Aldrich). After centrifugation at $14,000 \mathrm{~g}$ at $4^{\circ} \mathrm{C}$ for $20 \mathrm{~min}$, a total of $200 \mu \mathrm{g}$ of whole cell lysate was incubated with $1 \mu \mathrm{g}$ Flag antibodies (coupled with magnetic beads, L-1011, BioTNT, Shanghai, China) for $12 \mathrm{~h}$ at $4^{\circ} \mathrm{C}$. Next, the antibody, antigen, and magnetic bead complex were washed with IP/lysis buffer twice, boiled with 2x SDS-PAGE loading buffer (C508321, Sangon Biotech, Shanghai, China), and analyzed by western blot assay using corresponding antibodies. Normal mouse IgG was used as the negative control for immunoprecipitation.

\section{Dual-luciferase activity assay}

Ppara-driven promoter including three responsive elements (3x AGGACAAAGGTCA, PPRE ${ }_{3}$-TK-LUC), the Mlycd promoter (Mlycd-LUC) and the Gck promoter (Gck-LUC) was cloned into GV238 expression vectors. Renilla reporter plasmid was used as a negative control. HEK 293T cells were cultured in 48-well plates and transfected with $0.5 \mu \mathrm{g}$ corresponding plasmid and $0.05 \mu \mathrm{g}$ renilla vector for $6 \mathrm{~h}$. After $24 \mathrm{~h}$, luciferase activity in cultured cells was measured using the Dual-Luciferase@ Reporter assay system (E2920, Promega, Madison, WI) according to the manufacturer's instructions. Normalized firefly luciferase was obtained by dividing renilla luminescence units and was normalized to the ratio of the control group. Each experiment was performed with at least three samples for statistical significance.

Mitochondrial stress assay: Oxygen consumption rates were measured and calculated in the Seahorse XF24e analyzer. NRVMs were isolated and seeded into XF24e cell culture plates in a $2 \times 10^{4}$ density and allowed to adhere overnight. Then cells were transfected with lentivirus containing FABP3 or its negative virus and kept for 3 days for selection and treatment with Ang II for 24 hours before OCR measurement. 
One hour before the recording, the culture medium was replaced as Seahorse XF DMEM medium with 10 $\mathrm{mM}$ glucose, $2 \mathrm{mM}$ glutamine, $1 \mathrm{mM}$ pyruvate. Then OCR was recorded as subsequential injection of the following compounds: Oligomycin, 1.5 mM; FCCP, 3 mM; Rot/AA, 0.5 mM (103015-100, XF Cell Mito Stress Test Kit, Agilent Technologies).

LCFA oxidation stress assay: NRVMs were isolated as described before and 4,000 cells / well were seeded into Seahorse XF96 cell culture plate and cultured overnight. Next day, cells were transfected with lentivirus containing FABP3 or its negative virus for 24 hours and selected with puromycin for 3 days and treatment with Ang II for 24 hours before OCR measurement. One hour before the experiment, cell culture medium was changed to Seahorse XF DMEM medium with $10 \mathrm{mM}$ glucose, $2 \mathrm{mM}$ glutamine, $1 \mathrm{mM}$ pyruvate at a final $\mathrm{PH}$ of 7.4 and placed in a non-CO2 incubator for 1 hour. The following concentrations for each pot were applied in our assays: Etomoxir, 4 mM; Oligomycin, 1.5 mM; FCCP, 3 mM; Rot/AA, 0.5 mM (103672-100, XF Cell Mito Stress Test Kit, Agilent Technologies).

Glycolytic rate analysis: Real-time measurement of extracellular acidification rate (ECAR) and oxygen consumption rate (OCR) enables to determine of the glycolytic proton efflux rate (glycoPER) in detected cells. A total of 4,000 NRVMs were seeded into Seahorse XF96 cell culture plate and cultured overnight. Next day, cells were transfected with either FABP3 siRNA or FABP3 lentivirus and keep for 3 days. One hour before the experiment, cell culture medium was changed to Seahorse XF DMEM medium with 10 $\mathrm{mM}$ glucose, $2 \mathrm{mM}$ glutamine, $1 \mathrm{mM}$ pyruvate at a final $\mathrm{PH}$ of 7.4 and placed in a non-CO2 incubator for 1 hour. The following concentrations for each pot were applied in our assays: Rot/AA, $0.5 \mathrm{mM}$; 2-DG, 50 mM (103344-100, XF Cell Mito Stress Test Kit, Agilent Technologies).

\section{Statistical analysis}

Data are presented as the mean \pm SEM or mean \pm SD, which were performed using GraphPad Prism software (version 7.0a, San Diego, CA, USA) or Rstudio. The statistical differences between two groups were analyzed with two-sided Student's $t$-test. For experiments with more than two groups, after confirming normality and homogeneity of variance, one-way analysis of variance followed by Tukey's post-hoc test was used for comparison, otherwise, the Games-Howell post-hoc test was used.

Additionally, the Dunnett post-hoc test was applied to compare one group with the others. All statistical analyses were performed using SPSS software (version 23; SPSS Inc., Chicago, USA). To confirm the survival rate between WT and F3-KO mice, Kaplan-Meier survival curve and log-rank statistics tests were performed in Rstudio using the Survminer package. For all statistical analysis, $p$ value $<0.05$ was considered significant.

Data and materials availability: All relevant data are included in the figures, online figures, and Supplemental Materials. The scRNA-seq data presented in this article are available from the Gene Expression Omnibus (GEO) database under the accession number: GSE95143. Transcriptome data will be deposited in the GEO database upon acceptance of the article for publication. 


\section{Declarations}

\section{Competing interests:}

The authors declare no other competing interests.

\section{Author contributions:}

X.Y., C.K., and R.T. designed the study. L.Z., Z.L., and C.L. participated in the experimental design, data interpretation, manuscript preparation. Q.J., L.L., and K.C. discussed, edited and revised the manuscript.

\section{Acknowledgments}

This study was supported by the National Natural Science Foundation of China (81270004 to C.K.; 81970327 to R.T.; 81400362, 81670457, 81922007 and 91939103 to X.Y.), Shanghai Municipal Education Commission-Gaofeng Clinical Medicine Grant Support (20191803 to X.Y.), Shanghai Rising-Star Program grant (17QA1402300 to X.Y.), Municipal Human Resources Development Program for Outstanding Young Talents in Medical and Health Sciences in Shanghai (2017YQ017 to X.Y.), Shanghai Science and Technology Supporting Project (19411963400 to X.Y.), and a Natural Science Research Funding from Shanghai Jiao Tong University School of Medicine (19XJ11002 to X.Y.)

\section{References}

1. Collaborators GM. Global, regional, and national under-5 mortality, adult mortality, age-specific mortality, and life expectancy, 1970-2016: a systematic analysis for the Global Burden of Disease Study 2016 (vol 390, pg 1084, 2017). Lancet 390, E38-E38 (2017).

2. Nakamura M, Sadoshima J. Mechanisms of physiological and pathological cardiac hypertrophy. Nat Rev Cardiol 15, 387-407 (2018).

3. Shimizu I, Minamino T. Physiological and pathological cardiac hypertrophy. J Mol Cell Cardio/97, 245-262 (2016).

4. Wang $L$, et al. Single-cell reconstruction of the adult human heart during heart failure and recovery reveals the cellular landscape underlying cardiac function. Nat Cell Biol 22, 108-119 (2020).

5. Pascual F, Coleman RA. Fuel availability and fate in cardiac metabolism: A tale of two substrates. Bba-Mol Cell Biol L 1861, 1425-1433 (2016).

6. Lopaschuk GD, Ussher JR, Folmes CDL, Jaswal JS, Stanley WC. Myocardial Fatty Acid Metabolism in Health and Disease. Physiol Rev 90, 207-258 (2010).

7. Rijzewijk LJ, et al. Altered myocardial substrate metabolism and decreased diastolic function in nonischemic human diabetic cardiomyopathy: studies with cardiac positron emission tomography and magnetic resonance imaging. J Am Coll Cardiol 54, 1524-1532 (2009).

8. Ritterhoff J, et al. Metabolic Remodeling Promotes Cardiac Hypertrophy by Directing Glucose to Aspartate Biosynthesis. Circ Res 126, 182-196 (2020). 
9. Arumugam S, Sreedhar R, Thandavarayan RA, Karuppagounder V, Watanabe K. Targeting fatty acid metabolism in heart failure: is it a suitable therapeutic approach? Drug Discov Today 21, 1003-1008 (2016).

10. Lopaschuk GD, Ussher JR, Folmes CD, Jaswal JS, Stanley WC. Myocardial fatty acid metabolism in health and disease. Physiol Rev 90, 207-258 (2010).

11. Furuhashi M, Hotamisligil GS. Fatty acid-binding proteins: role in metabolic diseases and potential as drug targets. Nat Rev Drug Discov 7, 489-503 (2008).

12. Varrone F, et al. The Circulating Level of FABP3 Is an Indirect Biomarker of MicroRNA-1. J Am Coll Cardiol 61, 88-95 (2013).

13. Girard J, Duee P, Ferre P, Pegorier J, Escriva F, Decaux J. Fatty acid oxidation and ketogenesis during development. Reproduction Nutrition Développement 25, 303-319 (1985).

14. Vergnes L, Chin R, Young SG, Reue K. Heart-type fatty acid-binding protein is essential for efficient brown adipose tissue fatty acid oxidation and cold tolerance. J Biol Chem 286, 380-390 (2011).

15. Nomura $\mathrm{S}$, et al. Cardiomyocyte gene programs encoding morphological and functional signatures in cardiac hypertrophy and failure. Nat Commun 9, 4435 (2018).

16. Goldberg Ira J, Trent Chad M, Schulze PC. Lipid Metabolism and Toxicity in the Heart. Cell Metabolism 15, 805-812 (2012).

17. Barger PM, Brandt JM, Leone TC, Weinheimer CJ, Kelly DP. Deactivation of peroxisome proliferatoractivated receptor-alpha during cardiac hypertrophic growth. J Clin Invest 105, 1723-1730 (2000).

18. Liu F, et al. Upregulation of MG53 induces diabetic cardiomyopathy through transcriptional activation of peroxisome proliferation-activated receptor a. Circulation 131, 795-804 (2015).

19. Del Collado M, et al. Fatty Acid Binding Protein 3 And Transzonal Projections Are Involved In Lipid Accumulation During In Vitro Maturation Of Bovine Oocytes. Scientific reports 7, 2645 (2017).

20. Chen K, et al. Increment of HFABP Level in Coronary Artery In-Stent Restenosis Segments in Diabetic and Nondiabetic Minipigs: HFABP Overexpression Promotes Multiple Pathway-Related Inflammation, Growth and Migration in Human Vascular Smooth Muscle Cells. Journal of vascular research 53, 2738 (2016).

21. Zhuang LF, et al. Fatty acid-binding protein 3 contributes to ischemic heart injury by regulating cardiac myocyte apoptosis and MAPK pathways. Am J Physiol-Heart C 316, H971-H984 (2019).

22. Gerede DM, et al. Comparison of a qualitative measurement of heart-type fatty acid-binding protein with other cardiac markers as an early diagnostic marker in the diagnosis of non-ST-segment elevation myocardial infarction. Cardiovascular journal of Africa 26, 204-209 (2015).

23. Jacobs LH, et al. Rapidly rule out acute myocardial infarction by combining copeptin and heart-type fatty acid-binding protein with cardiac troponin. Annals of clinical biochemistry 52, 550-561 (2015).

24. Kagawa $Y$, et al. Comparison of heart-type fatty acid binding protein and sensitive troponin for the diagnosis of early acute myocardial infarction. Int J Cardio/ 166, 347-351 (2013). 
25. O'Donoghue M, et al. Prognostic utility of heart-type fatty acid binding protein in patients with acute coronary syndromes. Circulation 114, 550-557 (2006).

26. Kilcullen $\mathrm{N}$, et al. Heart-type fatty acid-binding protein predicts long-term mortality after acute coronary syndrome and identifies high-risk patients across the range of troponin values. Journal of the American College of Cardiology 50, 2061-2067 (2007).

27. DeLaughter DM, et al. Single-Cell Resolution of Temporal Gene Expression during Heart Development. Dev Cel/ 39, 480-490 (2016).

28. Vergnes L, Chin R, Young SG, Reue K. Heart-type fatty acid-binding protein is essential for efficient brown adipose tissue fatty acid oxidation and cold tolerance. J Biol Chem 286, 380-390 (2011).

29. Robinson E, Grieve DJ. Significance of peroxisome proliferator-activated receptors in the cardiovascular system in health and disease. Pharmacol Ther 122, 246-263 (2009).

30. Finck BN. The PPAR regulatory system in cardiac physiology and disease. Cardiovasc Res 73, 269277 (2007).

31. Feige JN, Gelman L, Michalik L, Desvergne B, Wahli W. From molecular action to physiological outputs: peroxisome proliferator-activated receptors are nuclear receptors at the crossroads of key cellular functions. Progress in lipid research 45, 120-159 (2006).

32. Burkart EM, et al. Nuclear receptors PPARbeta/delta and PPARalpha direct distinct metabolic regulatory programs in the mouse heart. J Clin Invest 117, 3930-3939 (2007).

33. Peters JM, et al. Alterations in lipoprotein metabolism in peroxisome proliferator-activated receptor adeficient mice. J Biol Chem 272, 27307-27312 (1997).

34. Lee $\mathrm{S}$, et al. Targeted disruption of the alpha isoform of the peroxisome proliferator-activated receptor gene in mice results in abolishment of the pleiotropic effects of peroxisome proliferators. Mol Cell Biol 15, 3012-3022 (1995).

35. Finck BN, et al. The cardiac phenotype induced by PPARa overexpression mimics that caused by diabetes mellitus. The Journal of clinical investigation 109, 121-130 (2002).

36. Hughes $\mathrm{ML}$, et al. Fatty acid-binding proteins 1 and 2 differentially modulate the activation of peroxisome proliferator-activated receptor a in a ligand-selective manner. J Biol Chem 290, 1389513906 (2015).

37. Velkov T. Interactions between human liver fatty acid binding protein and peroxisome proliferator activated receptor selective drugs. PPAR research 2013, (2013).

38. Hostetler HA, et al. L-FABP directly interacts with PPARa in cultured primary hepatocytes. Journal of lipid research 50, 1663-1675 (2009).

39. Tan NS, et al. Selective cooperation between fatty acid binding proteins and peroxisome proliferatoractivated receptors in regulating transcription. Mol Cell Biol 22, 5114-5127 (2002).

40. Zhang M, et al. CaMKII-delta9 promotes cardiomyopathy through disrupting UBE2T-dependent DNA repair. Nat Cell Biol 21, 1152-1163 (2019). 
41. Vogel B, Siebert H, Hofmann U, Frantz S. Determination of collagen content within picrosirius red stained paraffin-embedded tissue sections using fluorescence microscopy. MethodsX 2, 124-134 (2015).

42. Li R, Li Y, Kristiansen K, Wang J. SOAP: short oligonucleotide alignment program. Bioinformatics 24, 713-714 (2008).

43. Kim D, Langmead B, Salzberg SL. HISAT: a fast spliced aligner with low memory requirements. Nature methods 12, 357-360 (2015).

44. Dobin A, et al. STAR: ultrafast universal RNA-seq aligner. Bioinformatics 29, 15-21 (2013).

45. Love MI, Huber W, Anders S. Moderated estimation of fold change and dispersion for RNA-seq data with DESeq2. Genome biology 15, 550 (2014).

46. Subramanian A, et al. Gene set enrichment analysis: a knowledge-based approach for interpreting genome-wide expression profiles. Proceedings of the National Academy of Sciences 102, 1554515550 (2005).

47. Dunn WB, et al. Procedures for large-scale metabolic profiling of serum and plasma using gas chromatography and liquid chromatography coupled to mass spectrometry. Nature protocols 6,1060 (2011).

48. Trygg J, Wold S. Orthogonal projections to latent structures (O-PLS). Journal of Chemometrics: A Journal of the Chemometrics Society 16, 119-128 (2002).

49. Tsugawa H, Tsujimoto Y, Arita M, Bamba T, Fukusaki E. GC/MS based metabolomics: development of a data mining system for metabolite identification by using soft independent modeling of class analogy (SIMCA). BMC bioinformatics 12, 131 (2011).

\section{Figures}



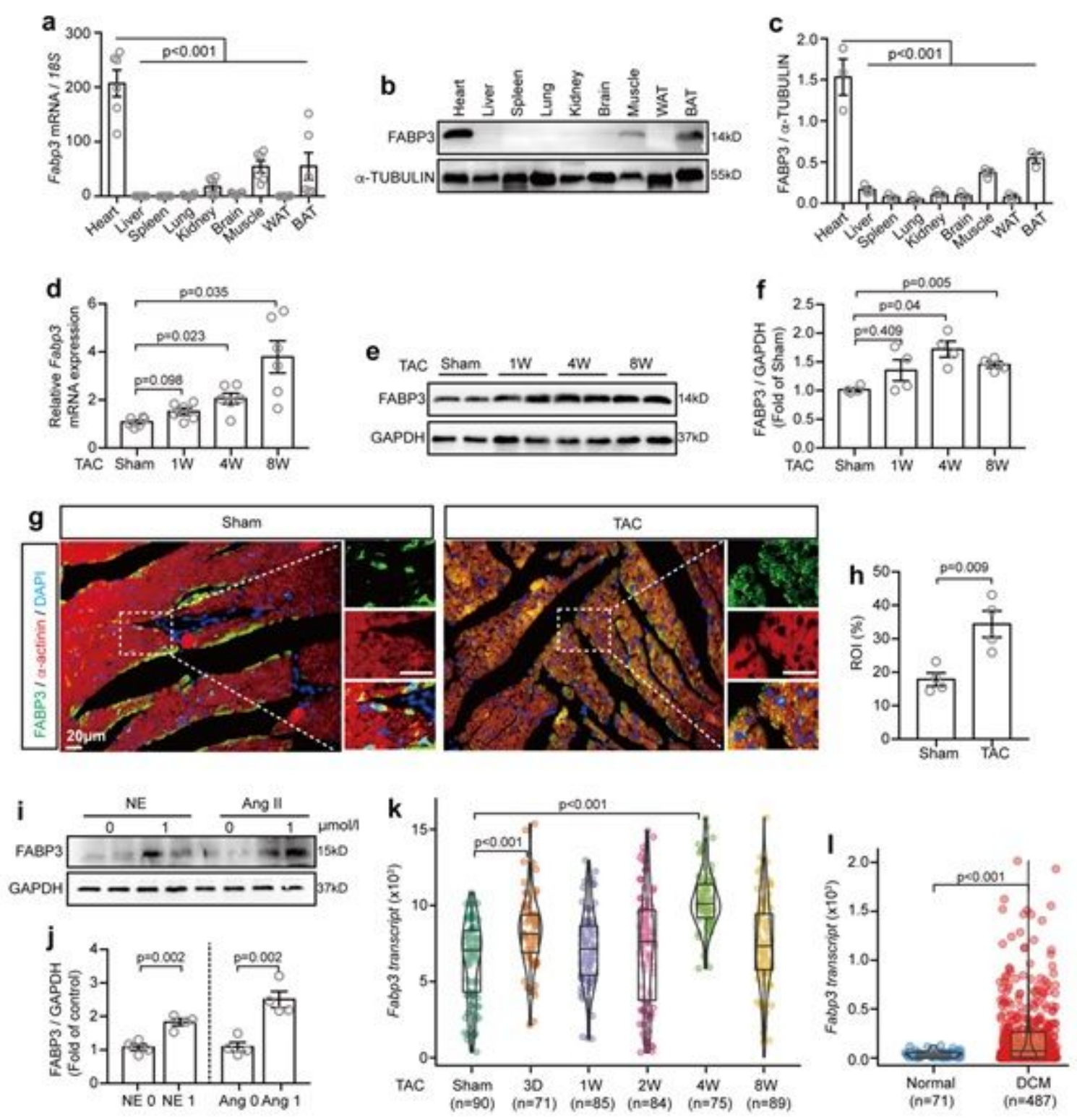

\section{Figure 1}

TAC or Ang II upregulates FABP3 expression in vivo and in vitro. a-c Mouse tissues were extracted for qPCR and western blot analyses to determine Fabp3 levels. a qPCR analysis of Fabp3 mRNA in the indicated organs; $18 \mathrm{~s}$ was used as the control. b FABP3 protein level in corresponding tissue were determined using western blotting. c Quantification of FABP3 expression in (b). d mRNA level of Fabp3 after TAC operation, as determined using QPCR. e Representative western blot images showing FABP3 expression. $\mathrm{f}$ Quantification results for (e). $\mathrm{g}$ Immunofluorescence co-staining of FABP3 (green) and aactinin (red) in sham- or TAC-operated heart sections. (Right) Higher magnification of dashed rectangle in left. h Percentage of FABP3+ cells in per region of interest (ROI) in (g). i NRVMs treated with NE or Ang II were subjected to western blotting assay to determine the FABP3 protein expression. j Quantification results of panel (i). $\mathrm{k}$-I Single cell RNA-sequencing of TAC-operated hearts or heart samples from DCM and normal patients were re-calculated for transcriptional expression of Fabp3 (GEO accession code: 
GSE95143). [a, $n=6 ; c, n=3 ;(a, c)$ : Dunnett's post-hoc test; $d, n=6 ; f, n=4 ;(d, f)$ : Games-Howell post-hoc test; $h, j, n=4 ;(h, j$, and I): Student's t-test; k: Dunnett's post-hoc test.]
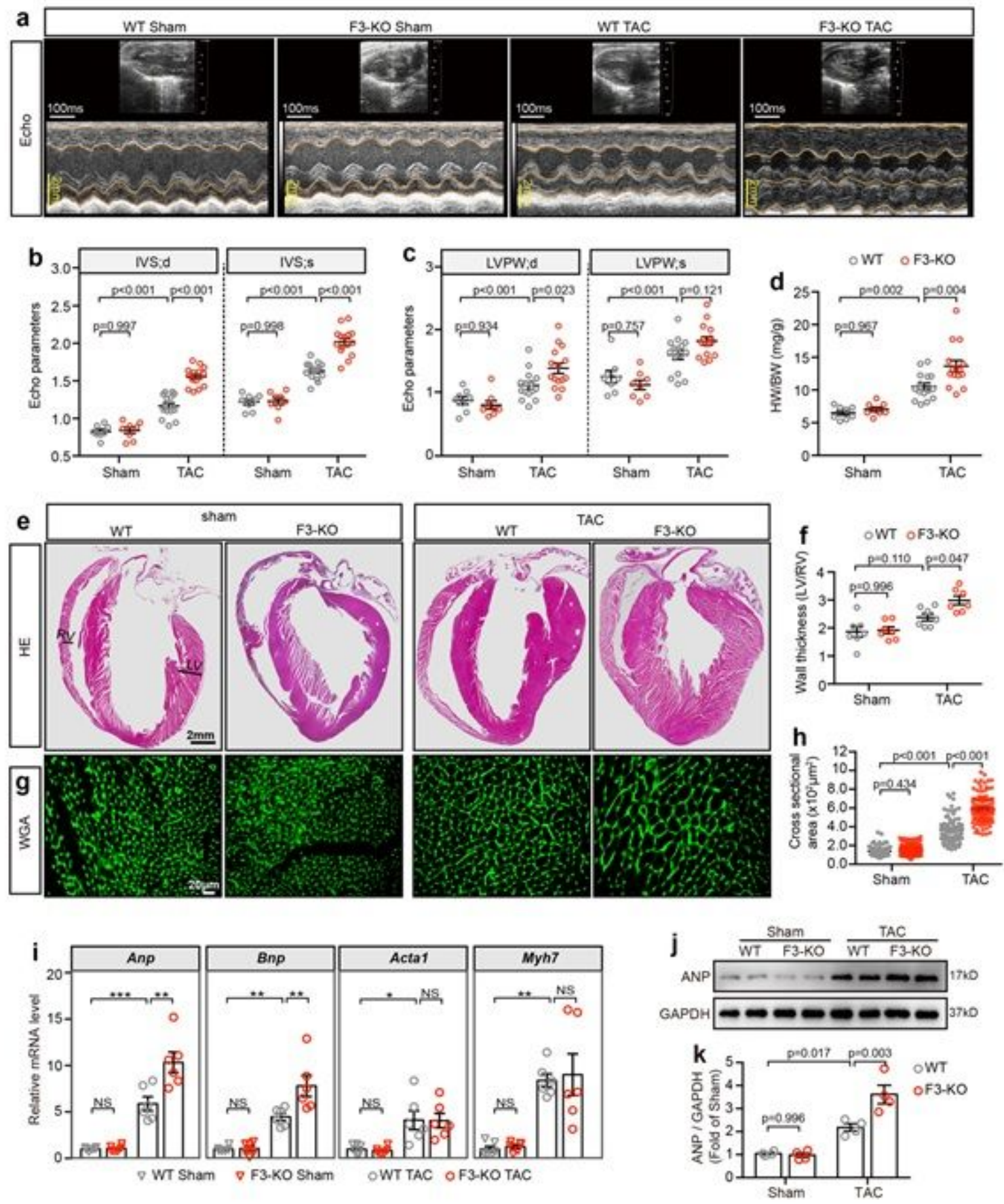

\section{Figure 2}

Deficiency of FABP3 aggravates TAC-induced cardiac hypertrophy. a Representative echo images of WT and F3-KO mice at 4 weeks after TAC or sham operation. b Quantification results for the interventricular septum (IVS) in panel (a). c Quantification results for the left ventricular posterior wall thickness (LVPW) in panel (a). d Ratio of heart weight to body weight from sham or TAC-operated WT or F3-KO mice. e Images of H\&E-stained longitudinal sections of the indicated hearts. f Quantification of wall thickness in (e), referred to the ratio of left ventricle (LV) to right ventricle (RV) length. g Heart sections stained with 
WGA to compare the size of cardiomyocyte area. h Quantification results in (g), a total of 100 cells /group was calculated. i qPCR assays compared the mRNA expression of Anp, Bnp, Acta1, and Myh7 at 4 weeks after sham or TAC operation. NS, not significant, ${ }^{*} p<0.05,{ }^{\star *} p<0.01$, ${ }^{\star \star \star} p<0.001$. j Representative western blot images of ANP in the indicated groups. $k$ Quantification results for (j). [b, $c, d, n=8,8,15,15$ respectively; $f, n=7 ; i, n=6 ; k, n=4 ;(b, c, d, f, h$, i and k): Tukey's post-hoc test.]
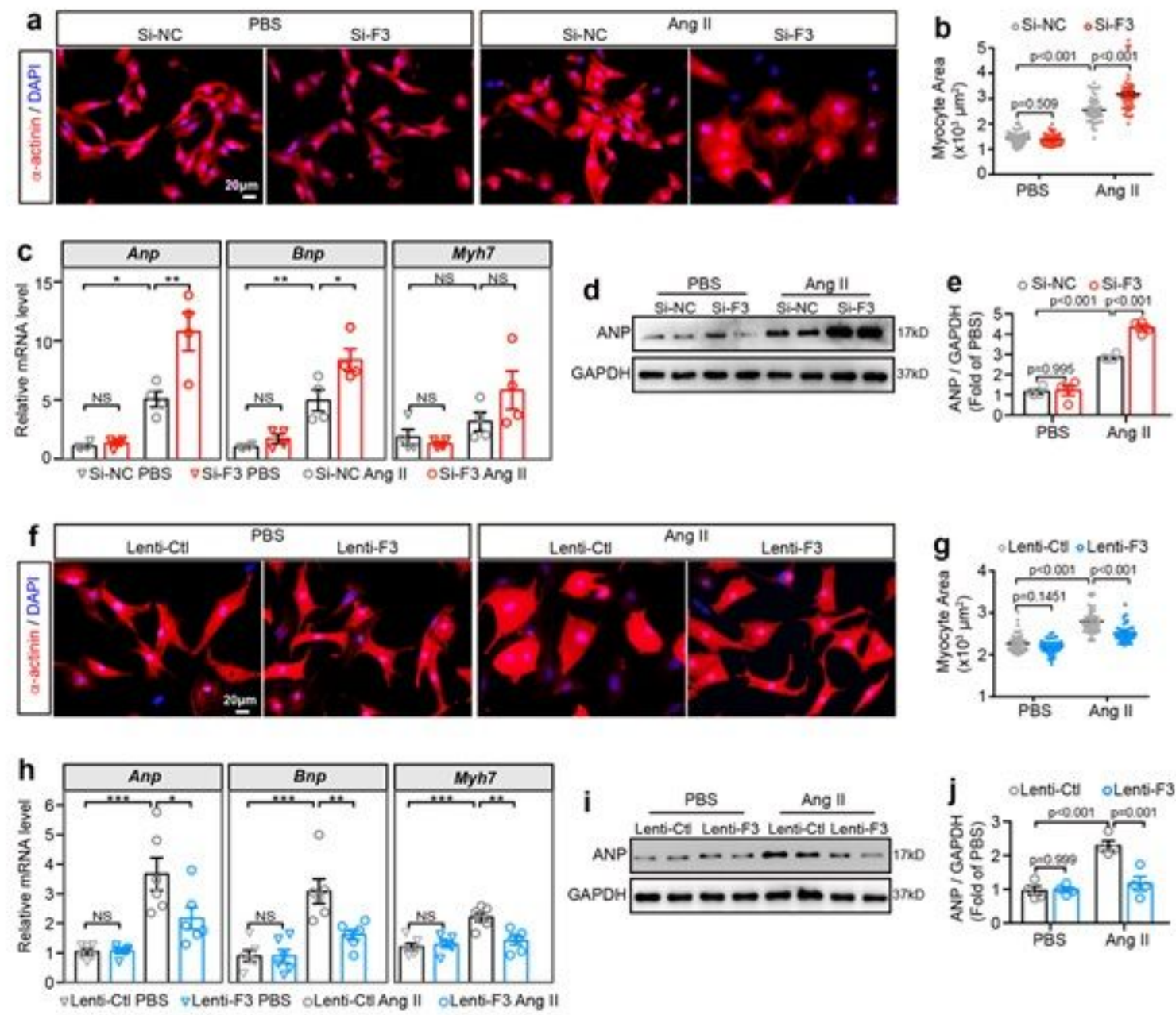

\section{Figure 3}

FABP3 participates in Ang II induced cell hypertrophy. a-e NRVMs were treated with siRNA-targeting Fabp3 (Si-F3) or its negative control (Si-NC) before PBS or Ang II treatment. a Immunofluorescence staining of a-actinin in NRVMs with or without Ang II treatment. b Quantification results of cell area in (a), 50 cells / group were calculated. c mRNA expression of hypertrophic genes (Anp, Bnp, and Myh7) in indicated groups. NS, not significant, $* p<0.05, * \star p<0.01$. d Representative western blot images showing increased ANP expression after Fabp3 knocking-down. e Quantification of panel (d). $f$-j NRVMs were transfected with lentivirus-encoding Fabp3 (Lenti-F3) or its negative control (Lenti-Ctl) before PBS or Ang II treatment. $f$ Cell area was determined by a-actinin staining in NRVMs. g Quantification results of cell area in (f), 50 cells / group were calculated. $h$ The mRNA levels of Anp, Bnp, and Myh7 were compared in the aforementioned groups. NS, not significant, ${ }^{*} p<0.05$, $* \star p<0.01, \star \star \star p<0.001$. i Representative 
western blot images revealed reduced ANP protein levels in the Lenti-F3 group. j Quantification results of (i). [c, e, j, n = 4; h, n = 6; (b, g): Games-Howell post-hoc test; (c, e, h, and j): Tukey's post-hoc test.]
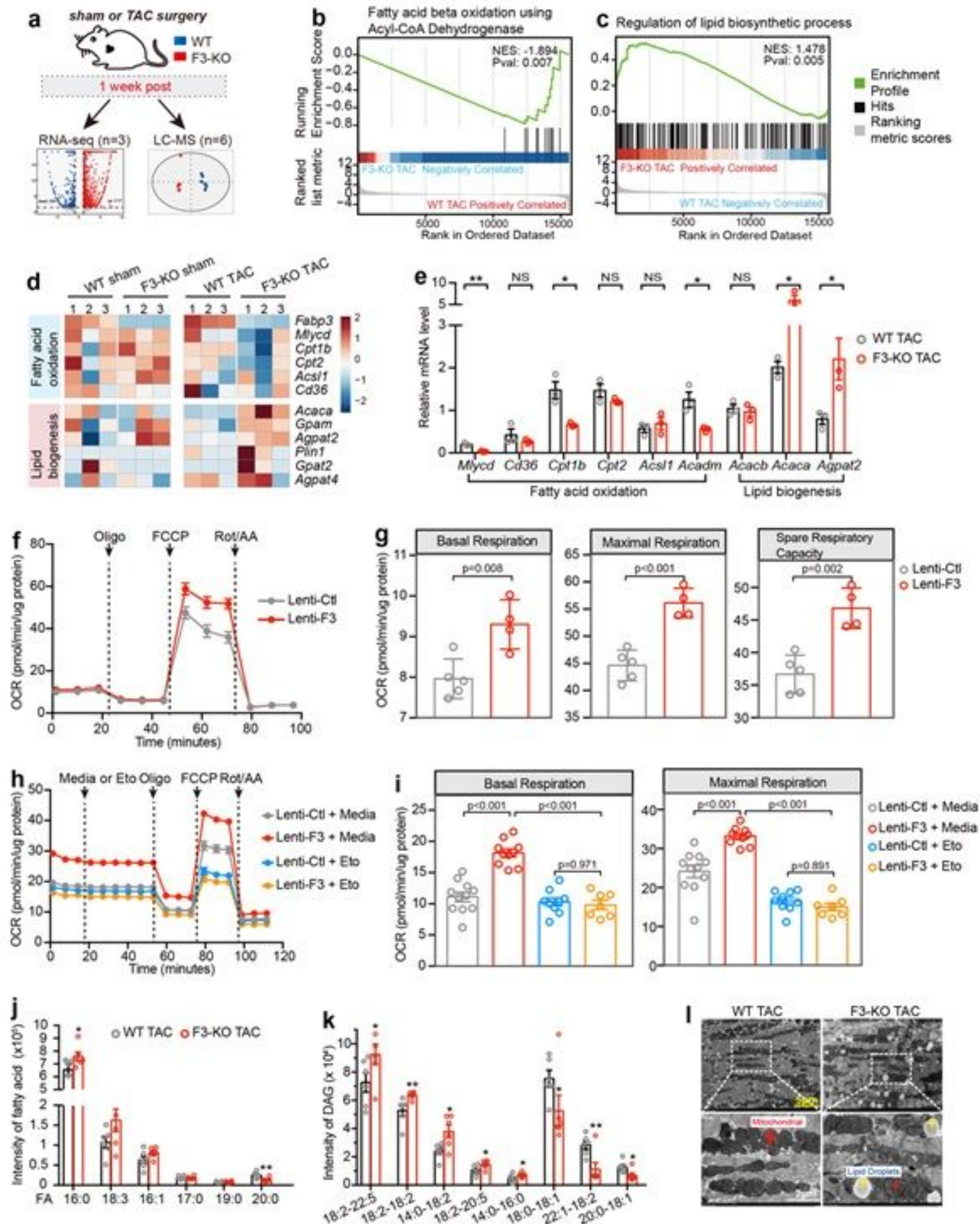

\section{Figure 4}

FABP3-defect results to compromised fatty acid oxidation (FAO) and toxic lipid accumulation. a Experimental schematic of RNA-seq analysis and metabolomics analysis in WT and F3-KO hearts. (b, c) GSEA analysis revealing that F3-KO negatively correlates with fatty acid beta oxidation using acyl-CoA dehydrogenase (b), while positively correlating with lipid biosynthetic process (c). d Heatmap showed 
scaled expression of FAO and lipid biogenesis genes in indicated samples from RNA-seq analysis. e mRNA expression of FAO and lipid biogenesis genes in TAC operated WT and F3-KO hearts. NS, not significant, ${ }^{*} p<0.05$, $* * p<0.01$. $f$ Mitochondrial stress assay was performed in NRVMs transfected with Fabp3 (Lenti-F3) or its negative control (Lenti-Ctl) after Ang II treatment to measure the oxygen consumption rates $(\mathrm{OCR})$, data was presented as mean \pm SD from individual experiments. $g$ The parameters of basal respiration, maximal respiration and spare respiration capacity were calculated from (f), data were presented as mean \pm SD from individual experiments. h LCFA oxidation stress assay was conducted in NRVMs with or without knocking-in expression of Fabp3 after Ang II treatment, etomoxir (Eto) was applied to inhibit the mitochondrial FAO. Data were presented as mean \pm SEM from individual experiments. $i$ The parameters of basal respiration, maximal respiration was calculated from $(h)$, data were presented as mean \pm SEM from individual experiments. $(j, k)$ The level of fatty acid (FA, j) and diacylglycerol (DAG, $\mathrm{k}$ ) in WT and F3-KO hearts determined by LC-MS analysis, 6 biological replicates / per group. ${ }^{*} p<0.05,{ }^{*} p<0.01$. I Representative electron micrographs of WT and F3-KO hearts after TAC operation. (Bottom) Higher magnification images of the dashed rectangle from (I). [e, $n=3$, Student's ttest; $\mathrm{g}, \mathrm{n}=5,4$, respectively, Student's t-test; $\mathrm{i}, \mathrm{n}=11,10,9,7$, respectively, Tukey's post-hoc test; (j, k): Student's t-test.] 

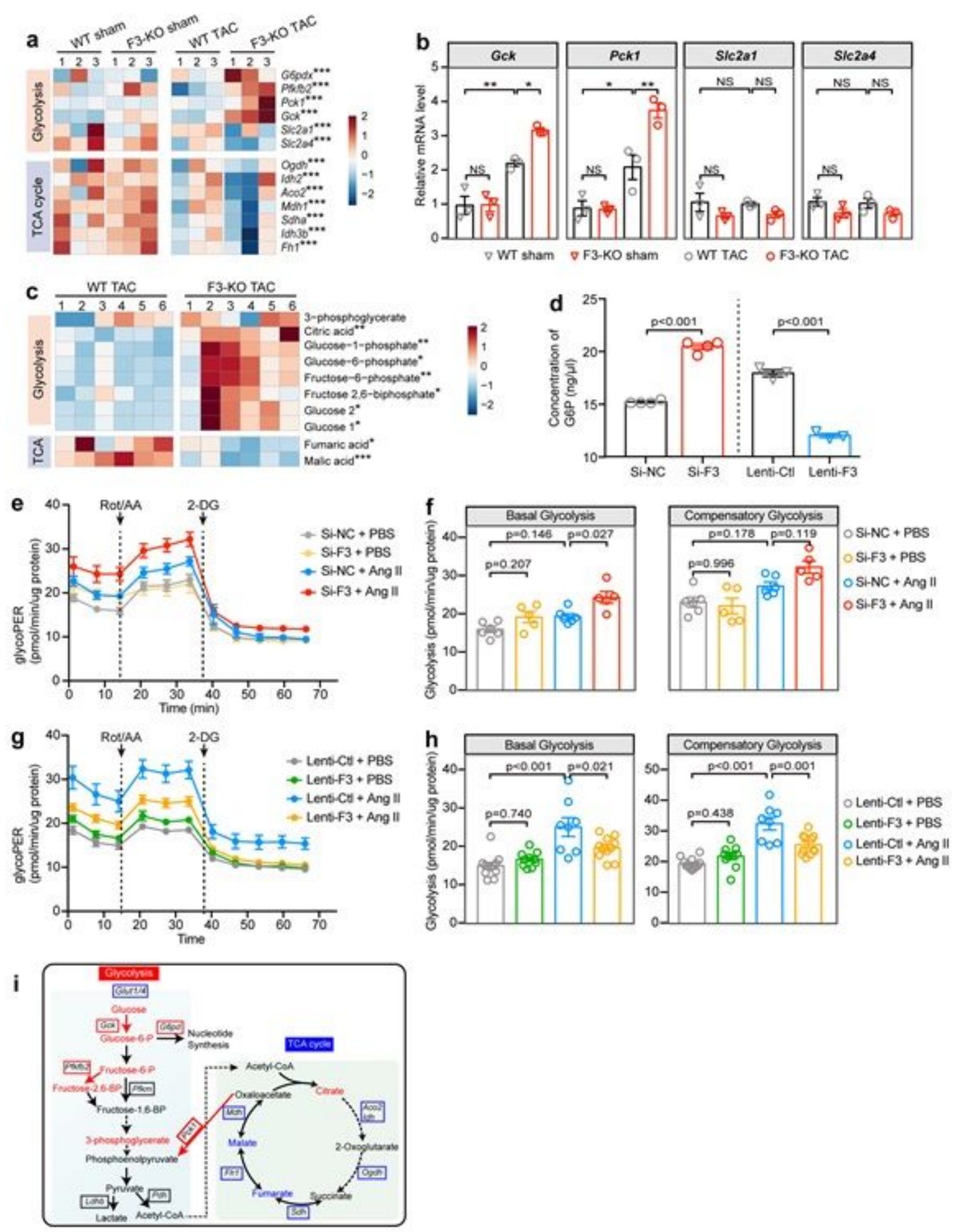

Figure 5

FABP3-null contributes to increased glycolysis and reduced ATP production under hypertrophic stimulation. a Heatmap of glycolysis and TCA cycle genes from RNA-seq results. b The mRNA expression of Gck, Pck1, Slc2a1, and Slc2a4 in indicated groups. NS, not significant, * $p<0.05$, ** $p<0.01$. c Heatmap of differential metabolites from WT and F3-KO hearts. ${ }^{*} p<0.05, * \star p<0.01, * \star * p<0.001$. d The intracellular concentration of glucose-6-phosphate (G6P) in NRVMs was measured after 
manipulating the expression of Fabp3. e Glycolytic rate assay was performed in NRVMs after knockingdown the expression of Fabp3 with siRNA to measure the OCR and extracellular acidification rates (ECAR) and converted to glycolytic proton efflux rate (glycoPER) in Seahorse Report Generator. $f$ The parameters of basal glycolysis and compensatory glycolysis were calculated from (e). Data were presented as mean \pm SEM from individual experiments. g Glycolytic rate assay was performed in NRVMs with or without Fabp3 knocking-in to measure glycoPER. h The parameters of basal glycolysis and compensatory glycolysis were calculated from $(\mathrm{g})$. Data were presented as mean \pm SD from individual experiments. i Schematic diagram including glycolysis/TCA cycle genes and metabolites. Red font and rectangles denote upregulated metabolites or genes, and blue represents those that are downregulated. $[b, n=3$, Tukey post-hoc test; $d, n=4,4,3,3$, respectively, Student's t-test; $f, n=6,5,6,5$, respectively; $h, n$ $=12,10,8,11$, respectively; $(f, h)$ : Tukey post-hoc test.] 

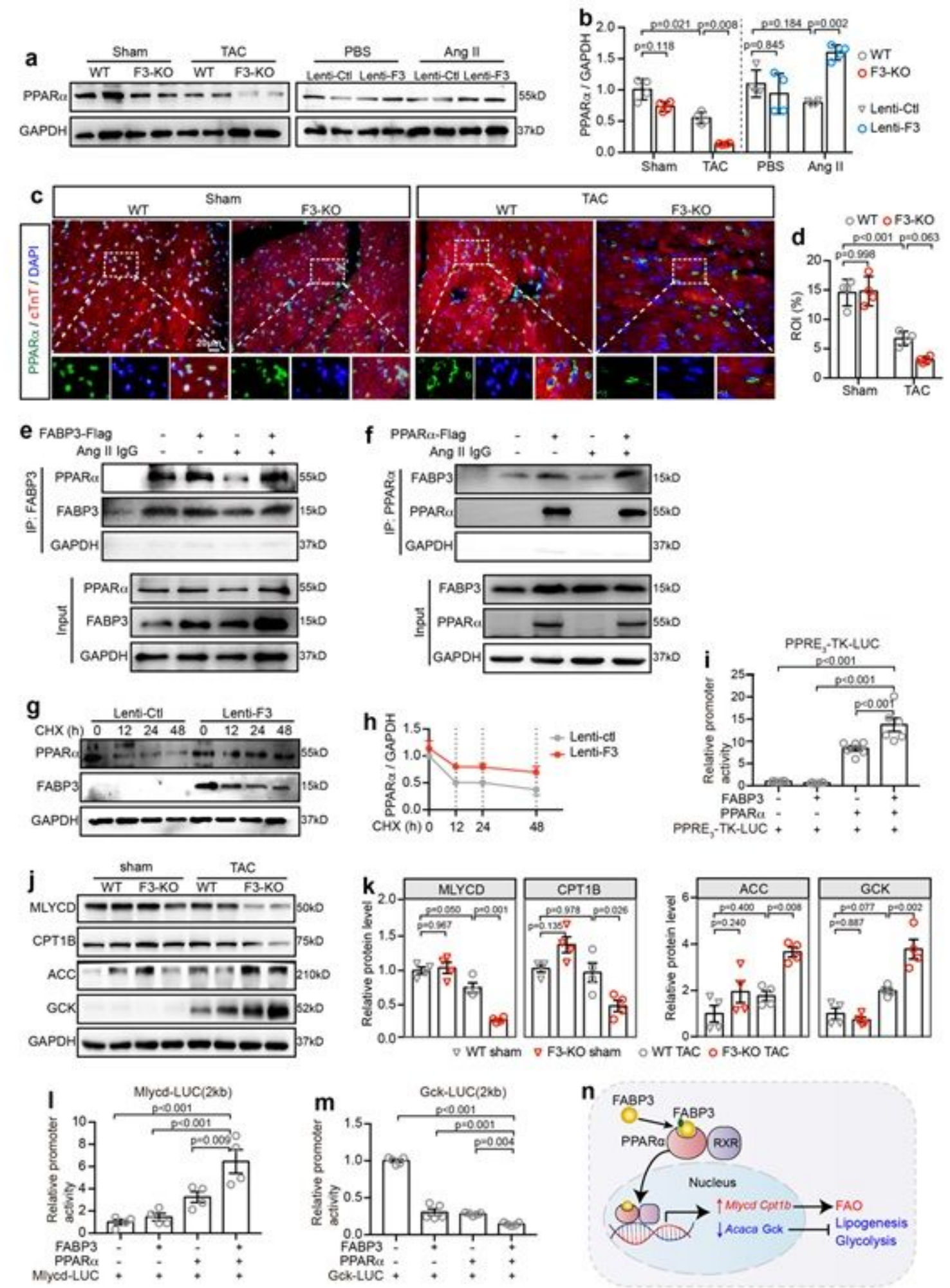

\section{Figure 6}

FABP3 mediates metabolic reprogramming by directly interacting with PPARa, preventing its degradation and enhancing its transactivation. a Representative western blot images of PPARa from TAC-operated WT and F3-KO hearts, or from NRVMs transfected with Fabp3 or its control virus with or without Ang II treatment. b Quantification of (a). c Immunofluorescence double-staining of PPARa (green) and cTnT (red) in WT and F3-KO hearts, with or without TAC surgery. (bottom) Higher magnification of dashed 
rectangle in the upper panel. d Quantification results in panel (c). e NRVMs with Fabp3 knocking-in were treated with or without Ang II for 24 hours, then co-immunoprecipitated (co-IP) FABP3. Western blotting assay was conducted with indicated antibodies. $f$ NRVMs with Ppar knocking-in were treated with or without Ang II for 24 hours, then co-immunoprecipitated (co-IP) of PPARa. g NRVMs transfected with Lenti-F3 or Lenti-Ctl were treated with $\mathrm{CHX}$ for $0,12,24$, or 48 hours to measure the protein expression of PPARa by western blotting. $h$ Quantification of PPARaintensities by normalizing to those of GAPDH in panel (g). i HEK 293T cells were transfected with PPRE-driven luciferase reporter (PPRE3-TK-LUC) and PPARa or FABP3 for 24 hours. Relative activation of PPRE3-TK-LUC as measured by normalizing its luminescence value to the renilla activity. $j$ Representative western blot images of MLYCD, CPT1B, ACC, GCK in WT and F3-KO mice after sham or TAC operation. $k$ The quantification results of panel $(\mathrm{j}) .(\mathrm{l}, \mathrm{m})$ HEK 293T cells were transfected with Mlycd-promoter luciferase reporter (Mlycd-LUC, I) or Gck-promoter luciferase reporter (Gck-Luc, m), PPARa or FABP3 as indicated for 24 hours. The relative expression of Mlycd-promoter and Gck-promoter was measured by normalizing their luminescence value to the corresponding renilla luminescence value. $n$ The schematic diagram shows FABP3 binds to PPARa and increases its transcriptional activity on Mlycd, Cpt1b while repressing Acaca, Gck to participate in cardiac FAO/glycolysis shift. [b, $n=4$, Games-Howell post-hoc test; $d, n=4$, Tukey's post-hoc test; $h, n=2 ; i, n=6$, Dunnett's post-hoc test; $k, n=4$, Tukey's post-hoc test; l, $n=4 ; m, n=5$; $(l, m)$ : Dunnett's post-hoc test.] 

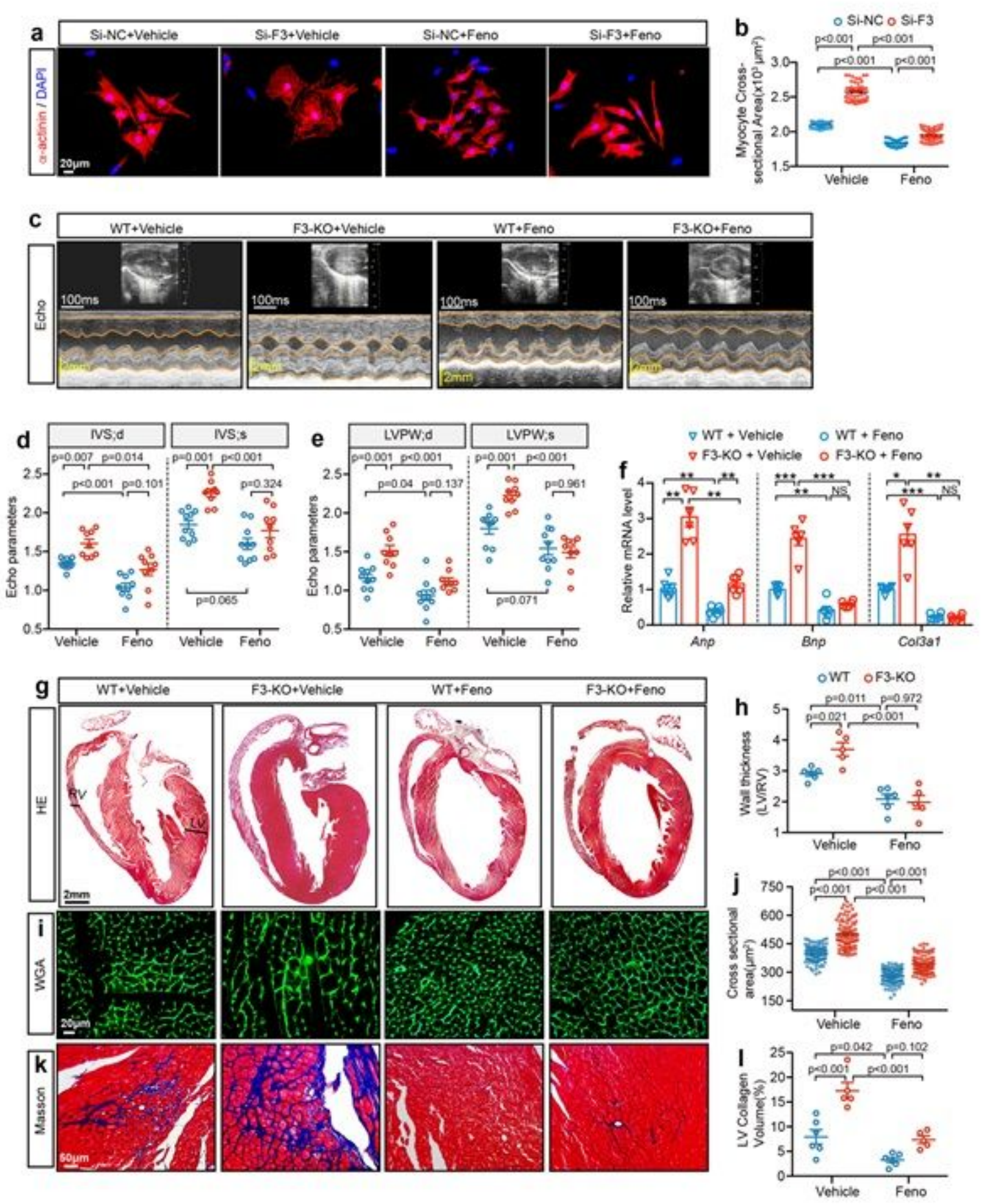

Figure 7

Fenofibrate represses FABP3-null induced cardiac hypertrophy in vivo and in vitro. a NRVMs treated with siRNA targeted Fabp3 (Si-F3) or its negative control before fenofibrate or vehicle treatment. Immunofluorescence staining of a-actinin used to compare myocyte area. b Quantification of panel (a). c Representative echo images in WT or F3-KO mice with or without fenofibrate treatment (Feno or Vehicle, respectively) at 4-weeks after TAC surgery. d Quantification of IVS in panel (c). e Quantification of LVPW in panel (c). $f$ Relative mRNA levels of Anp, Bnp, and Col3a1, as determined by qPCR in the indicated 
groups. NS, not significant, ${ }^{\star} p<0.05,{ }^{\star \star} p<0.01,{ }^{\star \star \star} p<0.001$. g Images of H\&E-stained longitudinal heart sections from WT or F3-KO mice with or without fenofibrate treatment. h Quantification of wall thickness in (g). i Representative immunofluorescence images of WGA staining from WT or F3-KO mice with or without fenofibrate treatment. j Quantification of cardiomyocyte cross-sectional area in (i) $(n=$ 100). $\mathrm{k}$ Masson staining to determine cardiac fibrosis in the aforementioned groups. I Quantification of collagen volume in (k). [d, e, $n=10,9,10,9$, respectively; (b, d-IVS; s): Games-Howell post hoc test; (d-IVS; d, e): Tukey's post-hoc test; $f, n=6$, (f-Anp, f-Col3a1, and j): Games-Howell post-hoc test; $h, I, n=6,5,6,5$; (f-Bnp, h, and I): Tukey's post-hoc test.]

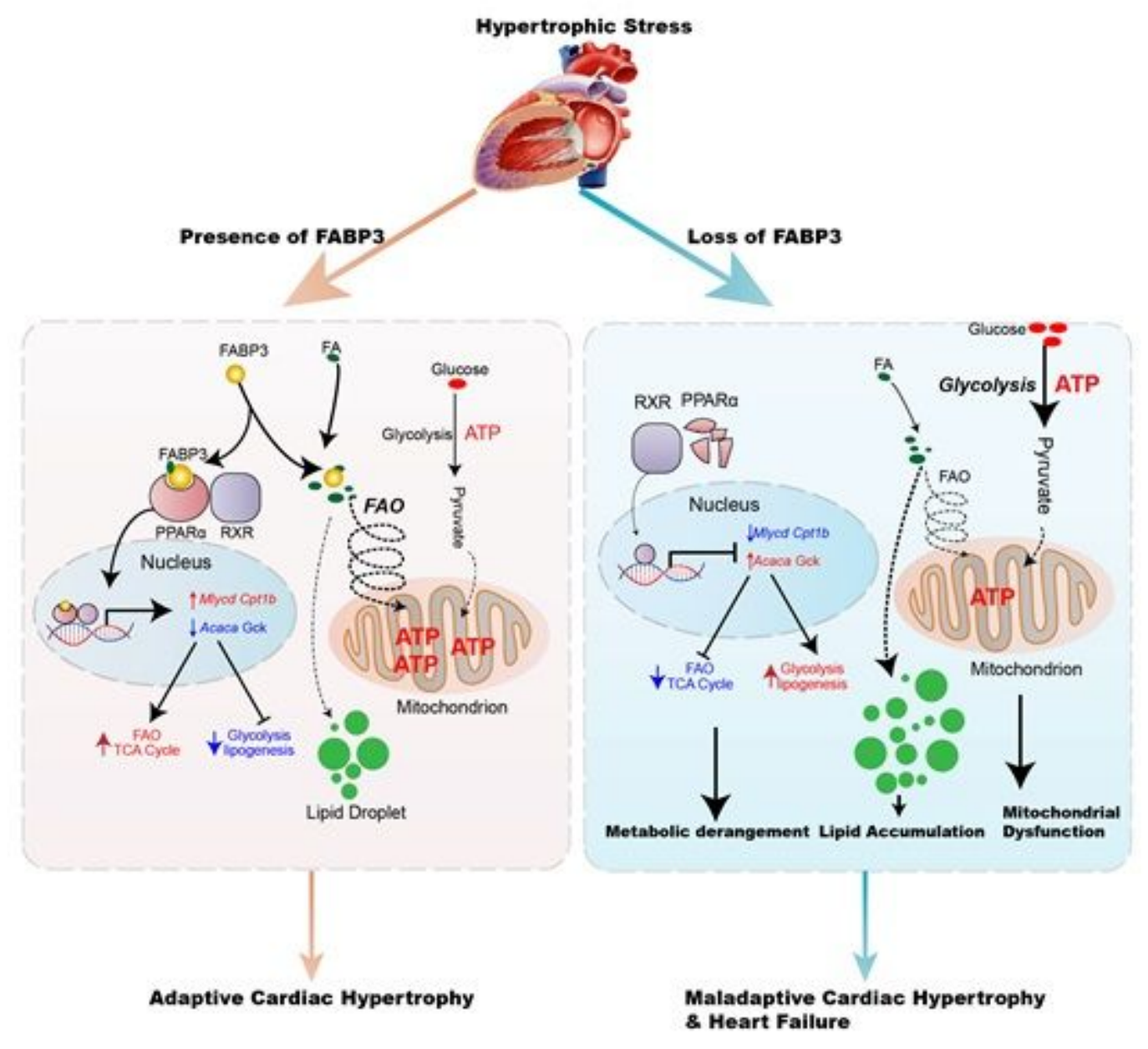

Figure 8

Model of FABP3-mediated PPARa pathway in cardiac metabolic reprogramming and progression of cardiac hypertrophy and heart failure. The schematic demonstrates that the FABP3-mediated PPARa pathway regulates cardiac metabolic reprogramming by directly interacting with PPARa, thereby stabilizing and inducing its transactivation which triggers downstream Mlycd and Cpt1b gene expression, while suppressing Acaca and Gck gene expression. This results in increased FAO levels and mitochondrional ATP production, ultimately resulting in adaptive cardiac function. However, in mice 
lacking FABP3, the response of FABP3-mediated PPARa on FAO/Glycolysis balance is abolished, leads to the degradation of PPARa, lower transcriptional activity on FAO genes accompanied by increased lipogenesis and glycolysis gene expression, which contributes to increase of glycolysis rate, accumulation of toxic lipid species, and compromised mitochondrial ATP production, thereby aggravating the progression of cardiac hypertrophy and heart failure.

\section{Supplementary Files}

This is a list of supplementary files associated with this preprint. Click to download.

- SUPPLEMENTALMATERIALS.pdf

- Imagesoffulluneditedgel.pdf 